\title{
ON THE BOUND STATES OF SCHRÖDINGER OPERATORS WITH $\delta$-INTERACTIONS ON CONICAL SURFACES
}

\author{
VLADIMIR LOTOREICHIK AND THOMAS OURMIÈRES-BONAFOS
}

\begin{abstract}
In dimension greater than or equal to three, we investigate the spectrum of a Schrödinger operator with a $\delta$-interaction supported on a cone whose cross section is the sphere of co-dimension two. After decomposing into fibers, we prove that there is discrete spectrum only in dimension three and that it is generated by the axisymmetric fiber. We get that these eigenvalues are non-decreasing functions of the aperture of the cone and we exhibit the precise logarithmic accumulation of the discrete spectrum below the threshold of the essential spectrum.
\end{abstract}

\section{INTRODUCTION}

1.1. Motivation. Some physical systems are efficiently described by Schrödinger operators with singular $\delta$-type interactions supported on various sets of zero Lebesgue measure (points, curves, surfaces or hypersurfaces). For instance, these operators are used to approximate atomic Hamiltonians in strong homogeneous magnetic fields [8] or photonic crystals with high-contrast [23]. The spectra of such Schrödinger operators are related to admissible values of the energy in quantum mechanics or, to admissible propagation frequencies of electromagnetic waves in optics.

A natural issue is to understand how the geometry of the support of the $\delta$-interaction influences the spectrum of these Schrödinger operators. This question is important, not only because of prospective applications in physics, but because it is also mathematically relevant in spectral geometry. For more information about this topic see the review paper [15], the monograph [19] and the references therein.

In dimension two, for $\delta$-interactions supported on curves, the question of finding a connection between the spectrum and the geometry was first addressed in [16]. In that paper, the authors consider two-dimensional Schrödinger operators with attractive $\delta$-interactions supported on asymptotically straight curves. Provided that the curve is not a straight line, they prove that there exists at least one bound state below the threshold of the essential spectrum. In the same spirit, we mention the special case of $\delta$-interactions supported on broken lines, investigated in [7, 14, 18, 20].

In dimension three the state of the art is not as complete as in dimension two. Instead of dealing with asymptotically straight curves, one is interested in attractive $\delta$-interactions supported on asymptotically flat surfaces and such Schrödinger operators were first studied in [17]. Provided the cross section is smooth, infinite conical surfaces give rise to a family of asymptotically flat surfaces. The special case of a circular cross section is investigated in [3] where the main result is the existence of infinitely many bound states below the threshold of the essential spectrum. Moreover, the authors bound from above the sequence of eigenvalues by a sequence which converges to the threshold of the essential spectrum at a known rate. Nevertheless, sharp spectral asymptotics on the number of eigenvalues remained unknown so far. We tackle this question in the present paper and give the precise rate of accumulation. It is reminiscent of [12], where the authors exhibit a similar result for a Dirichlet Laplacian in a conical layer. In this last paper, the authors also study the behaviour of the eigenvalues with respect to the aperture of the cone. Here, we restrain ourselves to show that the eigenvalues depend monotonously on the aperture of the cone.

In dimension greater than or equal to four very little is known so far. In the special case of an attractive $\delta$-interaction supported on a hyperconical surface we prove that there is no discrete spectrum. Because a hyperconical surface splits the Euclidean space into a convex domain and a non-convex conical domain, it is worth mentioning that this

2010 Mathematics Subject Classification. Primary 35P20; Secondary 35P15, 35Q40, 35Q60, $35 J 10$.

Key words and phrases. Schrödinger operator, $\delta$-interaction, existence of bound states, spectral asymptotics, conical and hyperconical surfaces. 
result strengthens the difference with Robin Laplacians in convex circular conical domains. Indeed, according to [28], these Robin Laplacians have infinite discrete spectrum for any dimension greater than or equal to three.

Finally, we emphasise that, for attractive $\delta$-interactions supported on conical surfaces, the structure of the essential spectrum strongly depends on the smoothness of the cross section. For attractive $\delta$-interactions supported on general non-smooth conical surfaces this structure is expected to be more involved and we refer to [5] for similar considerations about magnetic Laplacians on non-smooth conical domains.

1.2. Hamiltonians with $\delta$-interactions on conical surfaces. For $d \geq 3$, let $\left(x_{1}, x_{2}, x_{3}, \ldots, x_{d}\right)$ be the Cartesian coordinates on $\mathbb{R}^{d} .\left(L^{2}\left(\mathbb{R}^{d}\right) ;(\cdot, \cdot)_{\mathbb{R}^{d}}\right)$ and $\left(L^{2}\left(\mathbb{R}^{d} ; \mathbb{C}^{d}\right),(\cdot, \cdot)_{\mathbb{R}^{d}}\right)$ denote the usual $L^{2}$-space and the $L^{2}$-space of vector-valued functions over $\mathbb{R}^{d}$, respectively. The norms in $L^{2}\left(\mathbb{R}^{d}\right)$ and $L^{2}\left(\mathbb{R}^{d} ; \mathbb{C}^{d}\right)$ are both denoted by $\|\cdot\|_{\mathbb{R}^{d}}$ as there will be no danger of confusion. The first order $L^{2}$-based Sobolev space over $\mathbb{R}^{d}$ is denoted by $\left(H^{1}\left(\mathbb{R}^{d}\right)\right.$. We define the function $\mathbb{R}^{d} \ni x \mapsto \rho(x) \in \mathbb{R}_{+}$as

$$
\rho(x):=\sqrt{\sum_{k=1}^{d-1} x_{k}^{2}}
$$

and introduce the conical hypersurface $\mathcal{C}_{d, \theta} \subset \mathbb{R}^{d}$, as

$$
\mathcal{C}_{d, \theta}:=\left\{x=\left(x_{1}, x_{2}, \ldots, x_{d}\right) \in \mathbb{R}^{d}: x_{d}=(\cot \theta) \rho(x)\right\}, \quad \theta \in(0, \pi / 2) .
$$

The parameter $\theta$ is the half-opening angle (aperture) of the cone ( $c f$. Figure 1.1). Since there is no possible confusion, we denote the conical hypersurface by $\mathcal{C}$ instead of $\mathcal{C}_{d, \theta}$. We also denote by $\left.\left(L^{2}(\mathcal{C}) ;(\cdot, \cdot)_{\mathcal{C}}\right)\right)$ the $L^{2}$-space over $\mathcal{C}$.

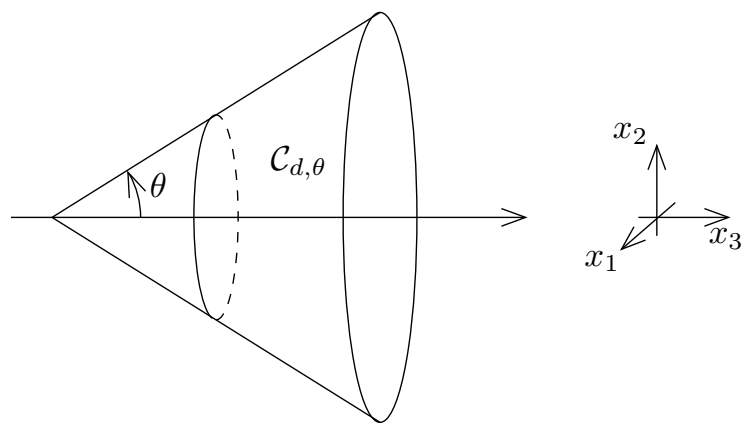

FIGURE 1.1. The cone $\mathcal{C}_{d, \theta}$ in dimension $d=3$.

For $\alpha>0$, we introduce the symmetric, densely defined sesquilinear form

$$
Q_{\alpha, \mathcal{C}}[u, v]:=(\nabla u, \nabla v)_{\mathbb{R}^{d}}-\alpha\left(\left.u\right|_{\mathcal{C}},\left.v\right|_{\mathcal{C}}\right)_{\mathcal{C}}, \quad \operatorname{dom} Q_{\alpha, \mathcal{C}}:=H^{1}\left(\mathbb{R}^{d}\right) .
$$

This form is closed and semibounded in $L^{2}\left(\mathbb{R}^{d}\right)$ (cf., e.g., [6, Sec. 2] and [2, Prop. 3.1]).

Definition 1.1. By the first representation theorem ([25, Ch. VI, Thm. 2.1]), the form $Q_{\alpha, \mathcal{C}}$ is associated with a self-adjoint operator $\mathrm{H}_{\alpha, \mathcal{C}}$ acting on $L^{2}\left(\mathbb{R}^{d}\right)$. This operator is called Schrödinger operator with $\delta$-interaction of strength $\alpha>0$ supported on $\mathcal{C}$.

The operator $\mathrm{H}_{\alpha, \mathcal{C}}$ can be understood as the Laplacian on $\mathbb{R}^{d}$ with a $\delta$-type coupling boundary condition on the conical surface $\mathcal{C}$. Formally, one can write $\mathrm{H}_{\alpha, \mathcal{C}}=-\Delta-\alpha \delta_{\mathcal{C}}$ or $\mathrm{H}_{\alpha, \mathcal{C}}=-\Delta-\alpha \delta(x-\mathcal{C})$. We refer to [2], for a rigorous description of the action of $\mathrm{H}_{\alpha, \mathcal{C}}$ and of its domain.

Before going any further, we remark that the conical structure of the system incites us to rescale the problem and to do so, we introduce the unitary transform, defined for all $u \in L^{2}\left(\mathbb{R}^{d}\right)$ by

$$
\left(\mathrm{U}_{\alpha} u\right)(x)=\alpha^{-d / 2} u\left(\alpha^{-1} x\right) .
$$

We have $\mathrm{H}_{\alpha, \mathcal{C}}=\alpha^{2} \mathrm{U}_{\alpha}^{-1} \mathrm{H}_{1, \mathcal{C}} \mathrm{U}_{\alpha}$ which reduces the study to the case $\alpha=1$. In the remaining part of this paper, we drop the index 1: $\mathrm{H}_{\mathcal{C}}$ and $Q_{\mathcal{C}}$ denote $\mathrm{H}_{1, \mathcal{C}}$ and $Q_{1, \mathcal{C}}$ respectively. 
1.3. Notations and main results. We introduce a few notation before stating the main results of this paper. The set of positive integers is denoted by $\mathbb{N}:=\{1,2, \ldots\}$ and the set of natural integers is denoted by $\mathbb{N}_{0}:=\mathbb{N} \cup\{0\}$. Let $\mathrm{T}$ be a semi-bounded self-adjoint operator associated with the quadratic form $\mathfrak{t}$. We denote by $\sigma_{\text {ess }}(\mathrm{T})$ and $\sigma_{\text {dis }}(\mathrm{T})$ the essential and the discrete spectrum of $\mathrm{T}$, respectively. By $\sigma(\mathrm{T})$, we denote the spectrum of $\mathrm{T}$ (i.e. $\sigma(\mathrm{T})=$ $\left.\sigma_{\text {ess }}(\mathbf{T}) \cup \sigma_{\text {dis }}(\mathbf{T})\right)$.

We set $E_{\text {ess }}(\mathrm{T}):=\inf \sigma_{\text {ess }}(\mathrm{T})$ and, for $k \in \mathbb{N}, E_{k}(\mathrm{~T})$ denotes the $k$-th eigenvalue of $\mathrm{T}$ in the interval $\left(-\infty, E_{\text {ess }}(\mathrm{T})\right)$. They are ordered non-decreasingly with multiplicities taken into account. We define the counting function of $T$ as

$$
\mathcal{N}_{E}(\mathrm{~T}):=\#\left\{k \in \mathbb{N}: E_{k}(\mathrm{~T})<E\right\}, \quad E \leq E_{\mathrm{ess}}(\mathrm{T}) .
$$

When working with the quadratic form $\mathfrak{t}$, we use the notations $\sigma_{\text {ess }}(\mathfrak{t}), \sigma_{\text {dis }}(\mathfrak{t}), \sigma(\mathfrak{t}), E_{\text {ess }}(\mathfrak{t}), E_{k}(\mathfrak{t})$ and $\mathcal{N}_{E}(\mathfrak{t})$ instead.

The first result is about the characterisation of the essential spectrum and the qualitative description of the discrete spectrum for $\mathrm{H}_{\mathcal{C}}$.

Theorem 1.2. Let $\theta \in(0, \pi / 2)$. The following statements hold:

(i) for any dimension $d \geq 3$, $\sigma_{\mathrm{ess}}\left(\mathrm{H}_{\mathcal{C}}\right)=[-1 / 4,+\infty)$;

(ii) for $d=3$, $\# \sigma_{\text {dis }}\left(\mathrm{H}_{\mathcal{C}}\right)=\infty$;

(iii) for $d \geq 4$, \# $\sigma_{\mathrm{dis}}\left(\mathrm{H}_{\mathcal{C}}\right)=0$.

Note that Theorem 1.2 was already known in dimension $d=3$ (cf. [3]). As the proof of the structure of the essential spectrum in dimension $d \geq 4$ follows exactly the same lines as the one exposed in [3, §2] (in dimension $d=3$ ), we omit it here for the sake of brevity. In fact, the result about the essential spectrum is obtained for a wide class of cones in any dimension in [9, Thm. 1.5]. The absence of discrete spectrum in item (iii) differs from the results in [16, 17] where, in dimension $d=2$ or $d=3$, it is shown that geometric deformations always induce bound states (at least if the interaction strength $\alpha>0$ is sufficiently large). To prove item (iii), we show that the operator $\mathrm{H}_{\mathcal{C}}$ is unitarily equivalent to an infinite orthogonal sum of self-adjoint fiber operators and that the spectrum of each fiber operator is included in $[-1 / 4,+\infty)$.

Then, relying on Theorem 1.2, we focus on properties of the discrete spectrum of $\mathrm{H}_{\mathcal{C}}$ in dimension $d=3$.

Proposition 1.3. In dimension $d=3$, for all $k \in \mathbb{N}$ the functions, $\theta \mapsto E_{k}\left(\mathrm{H}_{\mathcal{C}}\right)$ are non-decreasing on $(0, \pi / 2)$.

This proposition is reminiscent of a similar result in [20] for $\delta$-interactions supported on broken lines. Nevertheless, our proof is somewhat simpler since we do not use the Birman-Schwinger principle. The idea of the proof is to show that the discrete spectrum of $\mathrm{H}_{\mathcal{C}}$, below the point $-1 / 4$, coincides with the discrete spectrum of the lowest fiber operator below the same point. Then, one can show that the lowest fiber operator is unitarily equivalent to another operator, whose form domain is independent of $\theta$ and whose Rayleigh quotient is a monotone function of $\theta$.

Finally, we state our main result on the spectral asymptotics of $\mathrm{H}_{\mathcal{C}}$ for $d=3$.

Theorem 1.4. Let $\theta \in(0, \pi / 2)$. In dimension $d=3$, we have

$$
\mathcal{N}_{-1 / 4-E}\left(\mathrm{H}_{\mathcal{C}}\right) \sim \frac{\cot \theta}{4 \pi}|\ln (E)|, \quad E \rightarrow 0+.
$$

We want to emphasise the fact that thanks to unitary transform (1.4) and Theorem 1.4, we get for any $\alpha>0$ and $\theta \in(0, \pi / 2):$

$$
\mathcal{N}_{-\alpha^{2} / 4-E}\left(\mathrm{H}_{\alpha, \mathcal{C}}\right)=\mathcal{N}_{-1 / 4-\alpha^{-2} E}\left(\mathrm{H}_{\mathcal{C}}\right) \sim \frac{\cot \theta}{4 \pi}\left|\ln \left(\alpha^{-2} E\right)\right| \sim \frac{\cot \theta}{4 \pi}|\ln (E)|, \quad E \rightarrow 0+.
$$


The proof of Theorem 1.4 is inspired by a similar strategy developed in [12] for Dirichlet conical layers. Loosely speaking, we reduce the spectral asymptotics of $\mathrm{H}_{\mathcal{C}}$ to the known spectral asymptotics of the one-dimensional Schrödinger operator

$$
-\frac{\mathrm{d}^{2}}{\mathrm{~d} x^{2}}-\frac{1}{4 \sin ^{2} \theta} \frac{1}{x^{2}}, \quad \text { on }(1,+\infty)
$$

with Dirichlet or Neumann boundary condition at $x=1$. To this end, we use Dirichlet and Neumann bracketing combined with an IMS formula (cf. [10]). We emphasise that the geometry of the bracketings depends on the spectral parameter in a more sophisticated way than in [12]. Moreover, to estimate the operators involved in the Dirichlet and Neumann bracketings, we need spectral properties of two specific one-dimensional Schrödinger operators with a $\delta$-point interaction.

1.4. Discussion. Before going any further we now discuss open questions related to our results.

The strategy of our proofs heavily relies on the symmetry of the support of the $\delta$-interaction and it would be interesting to extend Theorem 1.4 to a more general class of cones. For instance, such structures are studied in [28] in another context.

We do not deal with the issue of existence/non-existence of eigenvalues embedded in the essential spectrum $[-1 / 4,+\infty)$. However, using the recent Rellich-type theorem for conical domains proven in [13] it is possible to exclude embedded eigenvalues in $[0,+\infty)$. Then, the study reduces to $[-1 / 4,0)$ and is still an open question.

Our knowledge on the eigenfunctions of $\mathrm{H}_{\mathcal{C}}$ is rather limited and we only prove that they are rotationally invariant. For Dirichlet conical layers, the numerical methods applied in $[12,21]$ suggest that the eigenfunctions corresponding to large eigenvalues look like the eigenfunctions of the one-dimensional operator (1.5). This is a direct consequence of the underlying tensored structure of the problem sufficiently far away from the vertex of the cone. In our case this tensored structure is lost and this is the reason why the proof of the spectral asymptotics is more sophisticated than in [12] for Dirichlet conical layers. In fact, we need to understand how far from the conical surface the eigenfunctions propagate and this is what the proof of Theorem 1.4 is about: We make use of Dirichlet and Neumann bracketing localised farther and farther away from the vertex of the cone. It implies that the spectral asymptotics is driven by the structure of the cone at infinity.

The small angle limit $\theta \rightarrow 0+$ could also be investigated by adapting the semi-classical methods used in [12]. It should yield localisation estimates for the eigenfunctions associated to the first eigenvalues as well as their asymptotic expansions with respect to the half-aperture of the cone $\theta$.

1.5. Organisation of the paper. In Section 2, we reduce the study of $\mathrm{H}_{\mathcal{C}}$ to a family of two-dimensional operators (fibers). This reduction allows to understand the structure of the discrete spectrum mentioned in Theorem 1.2 and to prove Proposition 1.3. After introducing one-dimensional model operators, Theorem 1.4 is proven in Section 3. Finally, we conclude the paper with Appendix A about some properties of fiber decompositions.

\section{FIBER DECOMPOSITION AND ITS FIRST APPLICATIONS}

In this section we prove Theorem 1.2 and Proposition 1.3. To take advantage of the symmetry of the problem, we work with a description of $\mathrm{H}_{\mathcal{C}}$ in cylindrical coordinates. Then, we reduce the study to a family of two-dimensional operators (fibers). For $d=3$, the fiber decomposition is also used in the proof of the spectral asymptotics in Section 3.

2.1. Hyper-cylindrical coordinates. Since the conical surface $\mathcal{C}$ is axisymmetric, the problem is better described in (hyper-)cylindrical coordinates with $x_{d}$ as the reference axis. Let us denote these coordinates by $(r, z, \phi) \in$ $\mathbb{R}_{+} \times \mathbb{R} \times \mathbb{S}^{d-2}$, where $\mathbb{S}^{d-2} \cong[0, \pi]^{d-3} \times[0,2 \pi)$ is the unit sphere of dimension $d-2$ and $\mathfrak{m}_{d-2}$ its natural surface measure. We can write $\phi \in \mathbb{S}^{d-2}$ as $\phi=\left(\phi_{1}, \ldots, \phi_{d-2}\right) \in[0, \pi]^{d-3} \times[0,2 \pi)$ and, for all $k \in\{1, \ldots, d-2\}$, we 
have

$$
x_{k}=r\left(\prod_{p=1}^{k-1} \sin \phi_{p}\right) \cos \phi_{k}, \quad x_{d-1}=r \prod_{p=1}^{d-2} \sin \phi_{p}, \quad x_{d}=z .
$$

For further use, we introduce the meridian domain $\mathbb{R}_{+}^{2}=\mathbb{R}_{+} \times \mathbb{R}$ and the meridian ray

$$
\Gamma_{\theta}:=\left\{(r, z) \in \mathbb{R}_{+}^{2}: z=r \cot \theta\right\},
$$

Now, we introduce some notations related to the cylindrical coordinates.

Notation 2.1. $L_{\text {cyl }}^{2}\left(\mathbb{R}^{d}\right)$ denotes the Hilbert space

$$
L_{\text {cyl }}^{2}\left(\mathbb{R}^{d}\right)=L^{2}\left(\mathbb{R}_{+}^{2} \times \mathbb{S}^{d-2}, r^{d-2} \mathrm{~d} r \mathrm{~d} z \mathrm{dm} \mathfrak{m}_{d-2}(\phi)\right) .
$$

Similarly, we define the Sobolev cylindrical space $H_{\mathrm{cyl}}^{1}\left(\mathbb{R}^{d}\right)$ by

$$
H_{\text {cyl }}^{1}\left(\mathbb{R}^{d}\right):=\left\{u \in L_{\text {cyl }}^{2}\left(\mathbb{R}^{d}\right): \partial_{r} u, \partial_{z} u, r^{-1}\left|\nabla_{\mathbb{S}^{d-2}} u\right| \in L_{\text {cyl }}^{2}\left(\mathbb{R}^{d}\right)\right\},
$$

endowed with the norm

$$
\|u\|_{H_{\text {cyl }}^{1}\left(\mathbb{R}^{d}\right)}^{2}:=\|u\|_{L_{\text {cyl }}^{2}\left(\mathbb{R}^{d}\right)}^{2}+\left\|\partial_{r} u\right\|_{L_{\text {cyl }}^{2}\left(\mathbb{R}^{d}\right)}^{2}+\left\|\partial_{z} u\right\|_{L_{\text {cyl }}^{2}\left(\mathbb{R}^{d}\right)}^{2}+\left\|r^{-1}\left|\nabla_{\mathbb{S}^{d-2}} u\right|\right\|_{L_{\text {cyl }}^{2}\left(\mathbb{R}^{d}\right)}^{2},
$$

where $\nabla_{\mathbb{S}^{d-2}}$ is the surface gradient on $\mathbb{S}^{d-2}$.

$\mathcal{C}_{0}^{\infty}\left(\overline{\mathbb{R}_{+}^{2}}\right)$ denotes the space of infinitely differentiable functions with compact support in $\overline{\mathbb{R}_{+}^{2}}$ and we introduce the space $\mathcal{C}_{0,0}^{\infty}\left(\overline{\mathbb{R}_{+}^{2}}\right)$ defined by

$$
\mathcal{C}_{0,0}^{\infty}\left(\overline{\mathbb{R}_{+}^{2}}\right):=\left\{u \in \mathcal{C}_{0}^{\infty}\left(\overline{\mathbb{R}_{+}^{2}}\right):\left.u\right|_{r=0}=0\right\}
$$

The change of variables (2.1) maps the whole space $\mathbb{R}^{d}$ onto $\mathbb{R}_{+}^{2} \times \mathbb{S}^{d-2}$ and $\mathrm{H}_{\mathcal{C}}$ becomes the unbounded self-adjoint operator $L_{\mathcal{C}}$ acting on $L_{\text {cyl }}^{2}\left(\mathbb{R}^{d}\right)$. By the first representation theorem, $L_{\mathcal{C}}$ can be seen as the operator associated with the quadratic form $Q_{\mathcal{C}}^{\text {cyl }}$, defined by the expression of $Q_{\mathcal{C}}$ in cylindrical coordinates:

$$
\begin{aligned}
& Q_{\mathcal{C}}^{\text {cyl }}[u]:=\left\|\partial_{r} u\right\|_{L_{\text {cyl }}^{2}\left(\mathbb{R}^{d}\right)}^{2}+\left\|\partial_{z} u\right\|_{L_{\text {cyl }}^{2}\left(\mathbb{R}^{d}\right)}^{2}+\left\|r^{-1}\left|\nabla_{\mathbb{S}^{d-2}} u\right|\right\|_{L_{\text {cyl }}^{2}\left(\mathbb{R}^{d}\right)}^{2} \\
&-\int_{\mathbb{R}_{+} \times \mathbb{S}^{d-2}}|u(s \sin \theta, s \cos \theta, \phi)|^{2}(s \sin \theta)^{d-2} \mathrm{~d} s \mathrm{dm}_{d-2}(\phi) . \\
& \operatorname{dom} Q_{\mathcal{C}}^{\text {cyl }}:=H_{\text {cyl }}^{1}\left(\mathbb{R}^{d}\right) .
\end{aligned}
$$

2.2. Spherical harmonics. First, we recall some known results on the spherical harmonics that can be found, e.g. , in [31, Chap. IV §2]. $-\Delta_{\mathbb{S}^{d-2}}$ denotes the Laplace-Beltrami on the sphere $\mathbb{S}^{d-2}$ and its eigenvalues are given by $l(l+d-3)$, with $l \in \mathbb{N}_{0}$ and the associated eigenspaces, denoted by $\mathcal{G}_{l}^{d-2}$, are of dimension

$$
c(d, l):=\left(\begin{array}{c}
d+l-2 \\
d-2
\end{array}\right)-\left(\begin{array}{c}
d+l-4 \\
d-2
\end{array}\right), \quad \text { where } \quad\left(\begin{array}{l}
n \\
k
\end{array}\right):=\left\{\begin{array}{cl}
\frac{n !}{k !(n-k) !} & \text { if } n \geq k, \\
0 & \text { otherwise. }
\end{array}\right.
$$

Moreover, for all $k \in\{1, \ldots, c(d, l)\}$, we denote by $Y_{l, k}^{d-2}$ the usual spherical harmonics. For all $l \in \mathbb{N}_{0}$ and $k \in\{1, \ldots, c(d, l)\}$, they satisfy

$$
-\Delta_{\mathbb{S}^{d-2}} Y_{l, k}^{d-2}=l(l+d-3) Y_{l, k}^{d-2}, \quad \mathcal{G}_{l}^{d-2}=\operatorname{span}_{k \in\{1, \ldots, c(d, l)\}}\left\{Y_{l, k}^{d-2}\right\} .
$$

Finally, the family of all the spherical harmonics forms an orthonormal basis of $\left(L^{2}\left(\mathbb{S}^{d-2}\right),\langle\cdot, \cdot\rangle_{\mathbb{S}^{d-2}}\right)$; i.e. ,

$$
\overline{\operatorname{span}_{l \in \mathbb{N}_{0}} \mathcal{G}_{l}^{d-2}}=L^{2}\left(\mathbb{S}^{d-2}\right)
$$


Now, decomposing into spherical harmonics and according to the terminology of [29, §XIII.16], we have the constant fiber sum

$$
\begin{aligned}
L^{2}\left(\mathbb{R}_{+}^{2} \times \mathbb{S}^{d-2}, r^{d-2} \mathrm{~d} r \mathrm{~d} z \mathrm{dm} \mathfrak{m}_{d-2}(\phi)\right) & =L^{2}\left(\mathbb{R}_{+}^{2}, r^{d-2} \mathrm{~d} r \mathrm{~d} z\right) \otimes L^{2}\left(\mathbb{S}^{d-2}\right) \\
& =\bigoplus_{l \in \mathbb{N}_{0}} \bigoplus_{k=1}^{c(d, l)} L^{2}\left(\mathbb{R}_{+}^{2}, r^{d-2} \mathrm{~d} r \mathrm{~d} z\right) .
\end{aligned}
$$

Secondly, for any function $u \in L_{\text {cyl }}^{2}\left(\mathbb{R}^{d}\right), l \in \mathbb{N}_{0}$ and $k \in\{1, \ldots, c(d, l)\}$ we define

$$
\left(\pi_{l, k} u\right)(r, z):=\left\langle u, Y_{l, k}^{d-2}\right\rangle_{\mathbb{S}^{d-2}}=\int_{\mathbb{S}^{d-2}} u(r, z, \phi) \overline{Y_{l, k}^{d-2}}(\phi) \mathrm{dm}_{d-2}(\phi)
$$

We consider the family $\left(\Pi_{l, k}\right)_{l \in \mathbb{N}_{0}, k \in\{1, \ldots, c(d, l)\}}$ of orthogonal projectors on $L_{\text {cyl }}^{2}\left(\mathbb{R}^{d}\right)$, defined as

$$
\left(\Pi_{l, k} u\right)(r, z, \phi):=\left(\pi_{l, k} u\right)(r, z) Y_{l, k}^{d-2}(\phi) .
$$

By definition of the spherical harmonics and $H_{\text {cyl }}^{1}\left(\mathbb{R}^{d}\right)$, we know that $\Pi_{l, k}\left(H_{\text {cyl }}^{1}\left(\mathbb{R}^{d}\right)\right) \subset H_{\text {cyl }}^{1}\left(\mathbb{R}^{d}\right)$. We introduce the quadratic forms

$$
Q_{\Gamma_{\theta}}^{[l, k]}[u]:=Q_{\mathcal{C}}^{\mathrm{cyl}}\left[u Y_{l, k}^{d-2}\right], \quad \operatorname{dom} Q_{\Gamma_{\theta}}^{[l, k]}:=\pi_{l, k}\left(H_{\mathrm{cyl}}^{1}\left(\mathbb{R}^{d}\right)\right) .
$$

By straightforward computations we first notice that the forms $Q_{\Gamma_{\theta}}^{[l, k]}$ do not depend on $k$ and to simplify, we drop the index $k$. Thirdly, we get

$$
\begin{gathered}
Q_{\Gamma_{\theta}}^{[l]}[u]=\int_{\mathbb{R}_{+}^{2}}\left(\left|\partial_{r} u\right|^{2}+\left|\partial_{z} u\right|^{2}\right. \\
\left.+\frac{l(l+d-3)}{r^{2}}|u|^{2}\right) r^{d-2} \mathrm{~d} r \mathrm{~d} z \\
-\int_{\mathbb{R}_{+}}|u(s \sin \theta, s \cos \theta)|^{2}(s \sin \theta)^{d-2} \mathrm{~d} s, \\
\operatorname{dom} Q_{\Gamma_{\theta}}^{[l]}= \begin{cases}\left\{u: u, \partial_{r} u, \partial_{z} u \in L^{2}\left(\mathbb{R}_{+}^{2}, r^{d-2} \mathrm{~d} r \mathrm{~d} z\right)\right\}, & l=0, \\
\left\{u: u, \partial_{r} u, \partial_{z} u, r^{-1} u \in L^{2}\left(\mathbb{R}_{+}^{2}, r^{d-2} \mathrm{~d} r \mathrm{~d} z\right)\right\}, & l \neq 0 .\end{cases}
\end{gathered}
$$

We refer to [4, §II.3.a] for a full description of the domains of the above forms when $d=3$.

Using (2.4), one can show that the quadratic forms $Q_{\Gamma_{\theta}}^{[l]}$ are symmetric, closed, densely defined and semibounded on $\pi_{l, k}\left(L_{\text {cyl }}^{2}\left(\mathbb{R}^{d}\right)\right)=L^{2}\left(\mathbb{R}_{+}^{2}, r^{d-2} \mathrm{~d} r \mathrm{~d} z\right)$. Hence, by the first representation theorem, each quadratic form $Q_{\Gamma_{\theta}}^{[l]}$ is associated with a self-adjoint operator $\mathrm{L}_{\Gamma_{\theta}}^{[l]}$ acting on $L^{2}\left(\mathbb{R}_{+}^{2}, r^{d-2} \mathrm{~d} r \mathrm{~d} z\right)$.

Using the precise description of dom $L_{\mathcal{C}}$ given in [2, Thm. 3.3 (a)] and the symmetry of $\mathcal{C}$, one can show that

$$
\Pi_{l, k}\left(\operatorname{dom} \mathrm{L}_{\mathcal{C}}\right) \subset \operatorname{dom} \mathrm{L}_{\mathcal{C}}, \quad \mathrm{L}_{\mathcal{C}}\left(\Pi_{l, k}\left(\operatorname{dom} \mathrm{L}_{\mathcal{C}}\right)\right) \subset \Pi_{l, k}\left(L_{\text {cyl }}^{2}\left(\mathbb{R}^{d}\right)\right) .
$$

The first representation theorem implies that the operator $\left.L_{\mathcal{C}}\right|_{\Pi_{l, k}\left(\operatorname{dom} L_{\mathcal{C}}\right)}$ on $\Pi_{l, k}\left(L_{\text {cyl }}^{2}\left(\mathbb{R}^{d}\right)\right)$ can be identified with $\mathrm{L}_{\Gamma_{\theta}}^{[l]}$ and that they have the same spectrum. By $[30, \S 1.4]$, the operator $\mathrm{L}_{\mathcal{C}}$ decomposes as

$$
\mathrm{L}_{\mathcal{C}}=\bigoplus_{l \in \mathbb{N}_{0}} \bigoplus_{k=1}^{c(d, l)} \mathrm{L}_{\Gamma_{\theta}}^{[l]}
$$

The self-adjoint operators $\mathrm{L}_{\Gamma_{\theta}}^{[l]}$ are the fibers of $\mathrm{L}_{\mathcal{C}}$ and this decomposition yields

$$
\sigma\left(\mathrm{H}_{\mathcal{C}}\right)=\sigma\left(\mathrm{L}_{\mathcal{C}}\right)=\overline{\cup_{l \in \mathbb{N}_{0}} \cup_{k=1}^{c(d, l)} \sigma\left(\mathrm{L}_{\Gamma_{\theta}}^{[l]}\right)}=\overline{\cup_{l \in \mathbb{N}_{0}} \sigma\left(\mathrm{L}_{\Gamma_{\theta}}^{[l]}\right)} .
$$

For further use, for all $d \geq 3$ and $l \geq 0$, we define $\operatorname{Co}\left(Q_{\Gamma_{\theta}}^{[l]}\right)$ as

$$
\operatorname{Co}\left(Q_{\Gamma_{\theta}}^{[l]}\right):= \begin{cases}\mathcal{C}_{0,0}^{\infty}\left(\overline{\mathbb{R}_{+}^{2}}\right), & \text { when }(d, l)=(3, l), \quad l>0 \\ \mathcal{C}_{0}^{\infty}\left(\overline{\mathbb{R}_{+}^{2}}\right), & \text { otherwise. }\end{cases}
$$

With this definition, $\operatorname{Co}\left(Q_{\Gamma_{\theta}}^{[l]}\right)$ is a form core of $Q_{\Gamma_{\theta}}^{[l]}(c f$. Proposition A.1). 
2.3. Flat metric. In this subsection, after reformulating the problem in the flat metric, we study the quadratic forms $Q_{\Gamma_{\theta}}^{[l]}$ with the help of a unitarily equivalent form. First, we formulate the following proposition.

Proposition 2.2. Let $\mathcal{H}$ and $\mathcal{G}$ be two Hilbert spaces and let $\mathrm{U}: \mathcal{H} \rightarrow \mathcal{G}$ be a unitary operator. Let $\mathfrak{s}$ be a closed, densely defined, symmetric and semibounded quadratic form on the Hilbert space $\mathcal{H}$. Define the quadratic form $\mathfrak{t}$ by

$$
\mathfrak{t}[u]:=\mathfrak{s}\left[\mathrm{U}^{-1} u\right], \quad \operatorname{dom} \mathfrak{t}:=\mathrm{U}(\operatorname{dom} \mathfrak{s}) .
$$

With this definition, the following statements hold.

(i) The form $\mathfrak{t}$ is closed, densely defined, symmetric, and semibounded on the Hilbert space $\mathcal{G}$; and we say that $\mathfrak{s}$ and $\mathfrak{t}$ are unitarily equivalent.

(ii) The respective self-adjoint operators $\mathrm{T}$ and $\mathrm{S}$ (associated with these forms) are unitarily equivalent and the relation $\mathrm{S}=\mathrm{U}^{-1} \mathrm{TU}$ holds.

(iii) If $\mathrm{Co}(\mathfrak{s})$ is a form core of $\mathfrak{s}$, then $\mathrm{U}(\mathrm{Co}(\mathfrak{s}))$ is a form core of $\mathfrak{t}$.

Proposition 2.2 is a direct consequence of the definition of $\mathfrak{t}$ and the definition of the unitary transform.

Secondly, we introduce the following unitary transform

$$
\mathrm{U}: L^{2}\left(\mathbb{R}_{+}^{2}, r^{d-2} \mathrm{~d} r \mathrm{~d} z\right) \rightarrow L^{2}\left(\mathbb{R}_{+}^{2}\right), \quad(\mathrm{U} u)(r, z):=r^{(d-2) / 2} u(r, z) \equiv \widetilde{u}(r, z) .
$$

Except for the axisymmetric fiber in dimension three, we are now able to reformulate the problem in the flat metric. The axisymmetric case in dimension three differs from the others because the functions in the form domain dom $Q_{\Gamma_{\theta}}^{[0]}$ satisfy a boundary conditions on the axis $r=0$ that behaves differently through the unitary transform (2.8). This is detailed right after the proof of the following proposition in Remark 2.4.

Proposition 2.3. Let $d \geq 3$ and $l \in \mathbb{N}_{0}$ be such that $(d, l) \neq(3,0)$. Then $Q_{\Gamma_{\theta}}^{[l]}$ is unitarily equivalent to the quadratic form

$$
\begin{aligned}
\widetilde{Q}_{\Gamma_{\theta}}^{[l]}[\widetilde{u}] & :=\int_{\mathbb{R}_{+}^{2}}\left(\left|\partial_{r} \widetilde{u}\right|^{2}+\left|\partial_{z} \widetilde{u}\right|^{2}+\frac{\gamma(d, l)}{r^{2}}|\widetilde{u}|^{2}\right) \mathrm{d} r \mathrm{~d} z-\int_{\mathbb{R}_{+}}|\widetilde{u}(s \sin \theta, s \cos \theta)|^{2} \mathrm{~d} s, \\
\operatorname{dom} \widetilde{Q}_{\Gamma_{\theta}}^{[l]} & :=\mathrm{U}\left(\operatorname{dom} Q_{\Gamma_{\theta}}^{[l]}\right),
\end{aligned}
$$

where $\gamma(d, l)=l(l+d-3)+\frac{1}{4}(d-2)(d-4) \geq 0$. Moreover, $\mathrm{U}\left(\operatorname{Co}\left(Q_{\Gamma_{\theta}}^{[l]}\right)\right)$ is a form core of $\widetilde{Q}_{\Gamma_{\theta}}^{[l]}$ and any function $\widetilde{u} \in \mathrm{U}\left(\mathrm{Co}\left(Q_{\Gamma_{\theta}}^{[l]}\right)\right)$ satisfies $\left.\widetilde{u}\right|_{r=0}=0$.

Proof. Let $(d, l) \neq(3,0)$. First, we define the quadratic form $\widetilde{Q}_{\Gamma_{\theta}}^{[l]}$ as

$$
\widetilde{Q}_{\Gamma_{\theta}}^{[l]}[\widetilde{u}]:=Q_{\Gamma_{\theta}}^{[l]}\left[\mathrm{U}^{-1} \widetilde{u}\right], \quad \operatorname{dom} \widetilde{Q}_{\Gamma_{\theta}}^{[l]}:=\mathrm{U}\left(\operatorname{dom} Q_{\Gamma_{\theta}}^{[l]}\right),
$$

with $U$ as in (2.8). By Proposition 2.2 (i) we know that $\widetilde{Q}_{\Gamma_{\theta}}^{[l]}$ is a closed, densely defined, symmetric, and semibounded quadratic form on $L^{2}\left(\mathbb{R}_{+}^{2}\right)$. To get the expression of $\widetilde{Q}_{\Gamma_{\theta}}^{[l]}$ stated in Proposition 2.3, it is sufficient to check it on a form core of $\widetilde{Q}_{\Gamma_{\theta}}^{[l]}$. Consequently, let us choose $u \in \operatorname{Co}\left(Q_{\Gamma_{\theta}}^{[l]}\right)$ and set $\widetilde{u}:=\mathrm{U} u$. Then, we have

$$
\begin{gathered}
\widetilde{Q}_{\Gamma_{\theta}}^{[l]}[\widetilde{u}]=Q_{\Gamma_{\theta}}^{[l]}\left[r^{-(d-2) / 2} \widetilde{u}\right]=\int_{\mathbb{R}_{+}^{2}}\left|\partial_{r}\left(r^{-(d-2) / 2} \widetilde{u}\right)\right|^{2} r^{d-2} \mathrm{~d} r \mathrm{~d} z+\int_{\mathbb{R}_{+}^{2}}\left(\left|\partial_{z} \widetilde{u}\right|^{2}+\frac{l(l+d-3)}{r^{2}}|\widetilde{u}|^{2}\right) \mathrm{d} r \mathrm{~d} z \\
-\int_{\mathbb{R}_{+}}|\widetilde{u}(s \sin \theta, s \cos \theta)|^{2} \mathrm{~d} s .
\end{gathered}
$$

A simple computation yields

$$
\left|\partial_{r} u\right|^{2}=\left|\partial_{r}\left(r^{-(d-2) / 2} \widetilde{u}\right)\right|^{2}=\frac{1}{r^{d-2}}\left|\partial_{r} \widetilde{u}\right|^{2}+\frac{(d-2)^{2}}{4 r^{d}}|\widetilde{u}|^{2}-\frac{d-2}{2 r^{d-1}} \partial_{r}\left(|\widetilde{u}|^{2}\right) .
$$


Integrating over $r \in \mathbb{R}_{+}$the last term in (2.9), and integrating by parts, we end up with

$$
\int_{\mathbb{R}_{+}} \frac{1}{r^{d-1}} \partial_{r}\left(|\widetilde{u}|^{2}\right) r^{d-2} \mathrm{~d} r=\int_{\mathbb{R}_{+}} \frac{1}{r} \partial_{r}\left(|\widetilde{u}|^{2}\right) \mathrm{d} r=\lim _{r \rightarrow+\infty}\left(r^{-1}|\widetilde{u}|^{2}\right)-\lim _{r \rightarrow 0}\left(r^{-1}|\widetilde{u}|^{2}\right)+\int_{\mathbb{R}_{+}} \frac{1}{r^{2}}|\widetilde{u}|^{2} \mathrm{~d} r .
$$

The two limits in (2.10) make sense and both are equal to zero. Indeed, we have $r^{-1}|\widetilde{u}|^{2}=|u|^{2} r^{d-3}$, and $u$ is compactly supported, so $|\widetilde{u}|^{2}=|u|^{2} r^{d-2} \rightarrow 0$ as $r \rightarrow+\infty$ and the first limit in (2.10) is zero. Now, when $d=3$, $u \in \mathcal{C}_{0,0}^{\infty}\left(\overline{\mathbb{R}_{+}^{2}}\right)$ so, $r^{-1}|\widetilde{u}|^{2}=|u|^{2} \rightarrow 0$ as $r \rightarrow 0$. When $d \geq 4, u \in \mathcal{C}_{0}^{\infty}\left(\overline{\mathbb{R}_{+}^{2}}\right)$ and $r^{-1}|\widetilde{u}|^{2}=|u|^{2} r^{d-3} \rightarrow 0$ as $r \rightarrow 0$. Hence, (2.10) rewrites as

$$
\int_{\mathbb{R}_{+}} \frac{1}{r^{d-1}} \partial_{r}\left(|\widetilde{u}|^{2}\right) r^{d-2} \mathrm{~d} r=\int_{\mathbb{R}_{+}} \frac{1}{r^{2}}|\widetilde{u}|^{2} \mathrm{~d} r .
$$

Using (2.9) and (2.11) we get the desired expression for $\widetilde{Q}_{\Gamma_{\theta}}^{[l]}$.

Remark 2.4. In the axisymmetric case in dimension three, which corresponds to $(d, l)=(3,0)$, we do not have $\lim _{r \rightarrow 0}\left(r^{-1}|\widetilde{u}|^{2}\right)=0$ in (2.10). Consequently, in the flat metric, the expression of $Q_{\Gamma_{\theta}}^{[0]}$ and the description of its form domain are more involved.

Now, we are ready to prove the following statement.

Proposition 2.5. Let $d \geq 3$ and $l \in \mathbb{N}_{0}$ be such that $(d, l) \neq(3,0)$. Then we have

$$
\inf \sigma\left(\mathrm{L}_{\Gamma_{\theta}}^{[l]}\right) \geq-1 / 4 \text {. }
$$

Proof. Instead of working with the operator $\mathrm{L}_{\Gamma_{\theta}}^{[l]}$ and its associated form $Q_{\Gamma_{\theta}}^{[l]}$ we work with the unitarily equivalent quadratic form in the flat metric $\widetilde{Q}_{\Gamma_{\theta}}^{[l]}$. We want to apply the min-max principle to the quadratic form $\widetilde{Q}_{\Gamma_{\theta}}^{[l]}$. To do so, it is sufficient to apply it with test functions in $\mathrm{U}\left(\operatorname{Co}\left(Q_{\Gamma_{\theta}}^{[l]}\right)\right)$, where $\mathrm{U}$ is as in (2.8). Let $\widetilde{u} \in \mathrm{U}\left(\operatorname{Co}\left(Q_{\Gamma_{\theta}}^{[l]}\right)\right)$, we have

$$
\widetilde{Q}_{\Gamma_{\theta}}^{[l]}[\widetilde{u}] \geq \int_{\mathbb{R}_{+}^{2}}\left(\left|\partial_{r} \widetilde{u}\right|^{2}+\left|\partial_{z} \widetilde{u}\right|^{2}\right) \mathrm{d} r \mathrm{~d} z-\int_{\mathbb{R}_{+}}|\widetilde{u}(s \sin \theta, s \cos \theta)|^{2} \mathrm{~d} s .
$$

Thanks to Proposition 2.3 we know that $\widetilde{u} \in H_{0}^{1}\left(\mathbb{R}_{+}^{2}\right)$. $\widetilde{u}$ can be extended by zero to the whole plane $\mathbb{R}^{2}$, defining a function $\widetilde{u}_{0} \in H^{1}\left(\mathbb{R}^{2}\right)$. We obtain

$$
\widetilde{Q}_{\Gamma_{\theta}}^{[l]}[\widetilde{u}] \geq \int_{\mathbb{R}^{2}}\left(\left|\partial_{r} \widetilde{u}_{0}\right|^{2}+\left|\partial_{z} \widetilde{u}_{0}\right|^{2}\right) \mathrm{d} r \mathrm{~d} z-\int_{\mathbb{R}}\left|\widetilde{u}_{0}(s \sin \theta, s \cos \theta)\right|^{2} \mathrm{~d} s .
$$

The quadratic form on the right-hand side is the one of a Schrödinger operator with an attractive $\delta$-interaction of strength 1 supported on a straight line in $\mathbb{R}^{2}$. Its spectrum can be computed via separation of variables and is $[-1 / 4,+\infty)$. The min-max principle applied to the form on the right hand side of (2.12) yields

$$
\widetilde{Q}_{\Gamma_{\theta}}^{[l]}[\widetilde{u}] \geq-(1 / 4)\left\|\widetilde{u}_{0}\right\|_{\mathbb{R}^{2}}^{2}=-(1 / 4)\|\widetilde{u}\|_{\mathbb{R}_{+}^{2}}^{2} .
$$

Finally, we get the inequality applying the min-max principle to $\widetilde{Q}_{\Gamma_{\theta}}^{[l]}$.

Remark 2.6. For two overlapping reasons, the argument used in the proof of the above proposition does not work for $(d, l)=(3,0)$. Firstly, we can not pass to the flat metric with Dirichlet boundary condition at $r=0$ as explained in Remark 2.4. Hence, the step of the argument based on the extension of the function $\widetilde{u}$ by zero, fails. Secondly, even if we find a way to address the problem in the flat metric with another boundary condition at $r=0$, the coefficient $\gamma(3,0)=-1 / 4$ is negative whereas for all $(d, l) \neq(3,0)$ holds $\gamma(d, l) \geq 0$. Thus, for $(d, l)=(3,0)$ the regular potential $\gamma(3,0) r^{-2}$ can not be dropped.

Combining the structure of the essential spectrum, stated in Theorem 1.2, with Proposition 2.5 we obtain the following corollary. 
Corollary 2.7. Let the self-adjoint operator $\mathrm{H}_{\mathcal{C}}$ be as in Definition 1.1 and the self-adjoint operator $\mathrm{L}_{\Gamma_{\theta}}^{[0]}$ be as in (2.6).

(i) For $d=3, \sigma_{\text {dis }}\left(\mathrm{H}_{\mathcal{C}}\right)=\sigma_{\operatorname{dis}}\left(\mathrm{L}_{\Gamma_{\theta}}^{[0]}\right)$ holds and the multiplicities of the corresponding eigenvalues coincide.

(ii) For $d \geq 4, \sigma_{\text {dis }}\left(\mathrm{H}_{\mathcal{C}}\right)=\varnothing$ holds.

In dimension $d \geq 4$, it proves Theorem 1.2 (iii) about the emptiness of $\sigma_{\text {dis }}\left(\mathrm{H}_{\mathcal{C}}\right)$. In dimension $d=3$, it reduces the study of the eigenvalues of $L_{\mathcal{C}}$ to its axisymmetric fiber $L_{\Gamma_{\theta}}^{[0]}$. In the remainder of this paper, except if stated explicitly, $d=3$ and to simplify the notations, we drop the index 0 and define

$$
\mathrm{L}_{\Gamma_{\theta}}:=\mathrm{L}_{\Gamma_{\theta}}^{[0]}, \quad Q_{\Gamma_{\theta}}:=Q_{\Gamma_{\theta}}^{[0]} .
$$

2.4. Monotonicity of the eigenvalues. We prove Proposition 1.3 about the monotonicity of the eigenvalues of $\mathrm{H}_{\mathcal{C}}$ with respect to the half-opening angle of the underlying cone $\mathcal{C}$. Thanks to Corollary 2.7 , we know that we only have to focus on the axisymmetric fiber $\mathrm{L}_{\Gamma_{\theta}}$. We describe the transition from the fiber form $Q_{\Gamma_{\theta}}$ (in (2.13)) on the meridian domain to a unitarily equivalent form on the inclined half-plane. This transition will be useful in the proof of Proposition 1.3 as well as in further considerations.

To this end, first, we define the rotation

$$
s=z \cos \theta+r \sin \theta, \quad t=-z \sin \theta+r \cos \theta,
$$

that transforms the meridian domain $\mathbb{R}_{+}^{2}$ into the inclined half-plane ( $c f$. Figure 2.1)

$$
\Omega_{\theta}:=\left\{(s, t) \in \mathbb{R}^{2}: s \sin \theta+t \cos \theta>0\right\} .
$$

The meridian ray $\Gamma_{\theta}$, defined in (2.2), becomes the ray

$$
\Gamma:=\left\{(s, 0) \in \Omega_{\theta}: s>0\right\} .
$$

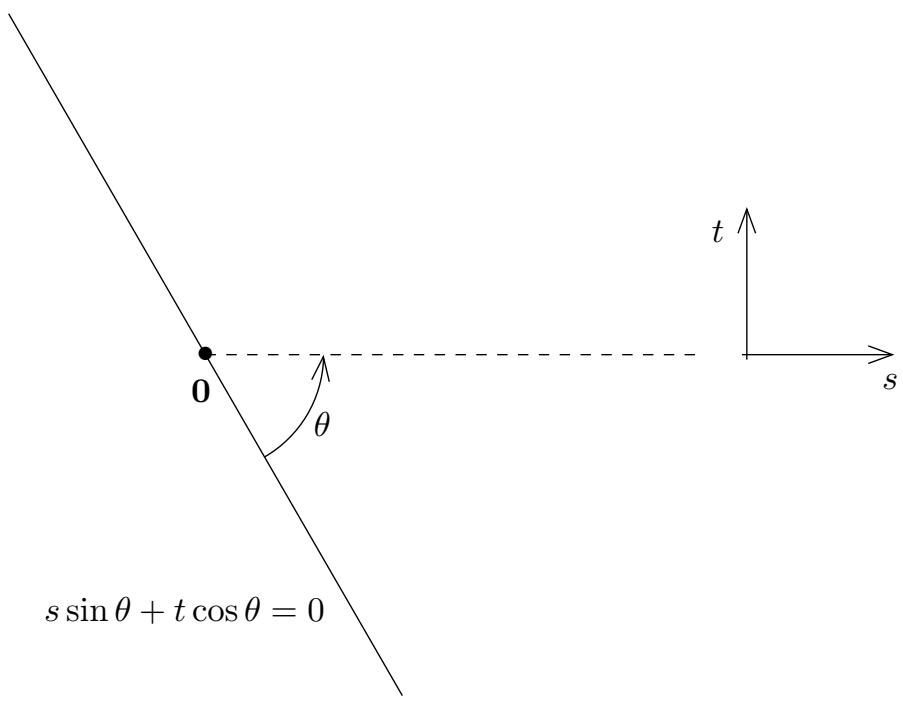

FIgURE 2.1. The inclined half-plane $\Omega_{\theta}$. The dashed line is the meridian ray $\Gamma$.

We associate the unitary operator

$$
\mathrm{U}: L^{2}\left(\mathbb{R}_{+}^{2}\right) \rightarrow L^{2}\left(\Omega_{\theta}\right), \quad(\mathrm{U} u)(s, t):=\widehat{u}(s, t)=u(s \sin \theta+t \cos \theta, s \cos \theta-t \sin \theta)
$$


with rotation (2.14). A straightforward computation yields that the quadratic form $Q_{\Gamma_{\theta}}$ defined in (2.13), is unitarily equivalent to $Q_{\Gamma}$, defined by

$$
\begin{aligned}
Q_{\Gamma}[\widehat{u}] & :=\int_{\Omega_{\theta}}\left(\left|\partial_{s} \widehat{u}\right|^{2}+\left|\partial_{t} \widehat{u}\right|^{2}\right)(s \sin \theta+t \cos \theta) \mathrm{d} s \mathrm{~d} t-\int_{\Gamma}|\widehat{u}(s, 0)|^{2} s \sin \theta \mathrm{d} s, \\
\operatorname{dom} Q_{\Gamma} & :=\mathrm{U}\left(\operatorname{dom} Q_{\Gamma_{\theta}}\right) .
\end{aligned}
$$

To avoid the dependence on $\theta$ of the domain $\operatorname{dom} Q_{\Gamma}$, we perform the change of variables $(s, t) \mapsto(\check{s}, \check{t})=(s \tan \theta, t)$ that transforms the domain $\Omega_{\theta}$ into $\Omega:=\Omega_{\pi / 4}$. Setting $\breve{u}(\breve{s}, \check{t})=\widehat{u}(s, t)$, for $u \in \operatorname{dom} Q_{\Gamma_{\theta}}$ we get, for the Rayleigh quotient

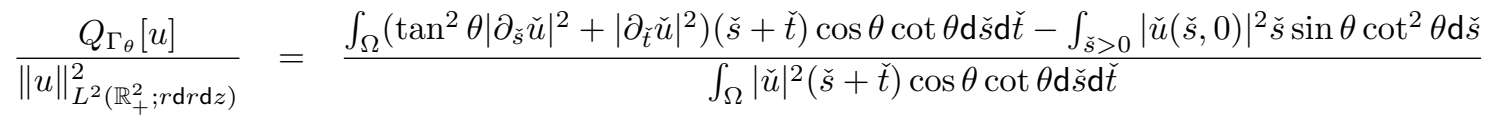

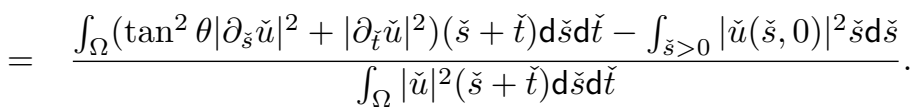

The right hand side of the last equation is the Rayleigh quotient of a quadratic form acting on $L^{2}(\Omega ;(\check{s}+\check{t}) \mathrm{d} \check{s} \mathrm{~d} \check{t})$. Because this form is unitarily equivalent to $Q_{\Gamma_{\theta}}$ and because its Rayleigh quotients are nondecreasing functions of $\theta$, the claim of Proposition 1.3 follows from the min-max formulae for the eigenvalues.

\section{Spectral asymptotics of $\mathrm{H}_{\mathcal{C}}$ In the threE-Dimensional CASE}

In this section we prove Theorem 1.4. The idea is to exhibit a lower and an upper bound for the counting function of the operator $\mathrm{L}_{\Gamma_{\theta}}$. We recall that all along this section $d=3$.

3.1. Proof of Theorem 1.4. The proof of Theorem 1.4 relies on the following two propositions, whose proofs are postponed to Subsections 3.3 and 3.4, respectively.

Proposition 3.1. Let $\theta \in(0, \pi / 2)$. We have

$$
\liminf _{E \rightarrow 0+} \frac{\mathcal{N}_{-1 / 4-E}\left(\mathrm{H}_{\mathcal{C}}\right)}{|\ln E|} \geq \frac{\cot \theta}{4 \pi} .
$$

Proposition 3.2. Let $\theta \in(0, \pi / 2)$. We have

$$
\limsup _{E \rightarrow 0+} \frac{\mathcal{N}_{-1 / 4-E}\left(\mathrm{H}_{\mathcal{C}}\right)}{|\ln E|} \leq \frac{\cot \theta}{4 \pi} .
$$

Propositions 3.1 and 3.2 imply

$$
\lim _{E \rightarrow 0+} \frac{\mathcal{N}_{-1 / 4-E}\left(\mathrm{H}_{\mathcal{C}}\right)}{|\ln E|}=\frac{\cot \theta}{4 \pi},
$$

which proves Theorem 1.4.

3.2. Auxiliary one-dimensional operators. In this subsection we discuss some spectral properties of one-dimensional model Schrödinger operators, which are used in the proofs of Propositions 3.1 and 3.2.

Let us start by studying two Schrödinger operators with a point $\delta$-interaction. For the spectral theory of onedimensional Schrödinger operators with point $\delta$-interactions we refer to [1, Chs. I.3, II.2, III.2], the review paper [27] and the references therein.

For $L>0$, we define the interval $I:=(-L, L)$ and introduce the Hilbert space $\left(L^{2}(I),(\cdot, \cdot) I\right)$. Let us consider the following two symmetric sesquilinear forms

$$
\begin{array}{ll}
\mathfrak{q}_{L}^{\mathrm{D}}[\varphi, \psi]:=\left(\varphi^{\prime}, \psi^{\prime}\right)_{I}-\varphi(0) \overline{\psi(0)}, & \operatorname{dom} \mathfrak{q}_{L}^{\mathrm{D}}:=H_{0}^{1}(I), \\
\mathfrak{q}_{L}^{\mathrm{N}}[\varphi, \psi]:=\left(\varphi^{\prime}, \psi^{\prime}\right)_{I}-\varphi(0) \overline{\psi(0)}, & \operatorname{dom} \mathfrak{q}_{L}^{\mathrm{N}}:=H^{1}(I),
\end{array}
$$


one can verify that both forms are closed, densely defined, and semibounded in $L^{2}(I)$. For $\varphi \in H^{2}(I \backslash\{0\})$ set $\varphi_{+}:=\left.\varphi\right|_{(0, L)}, \varphi_{-}:=\left.\varphi\right|_{(-L, 0)}$ and $\left[\varphi^{\prime}\right](0):=\varphi^{\prime}(0+)-\varphi^{\prime}(0-)$. Thanks to the first representation theorem ([25, Ch. VI, Thm. 2.1]), each of these quadratic forms is associated with a unique self-adjoint operator acting on $L^{2}(I)$. The respective operators are given by

$$
\begin{aligned}
& \mathrm{h}_{L}^{\mathrm{D}} \varphi=-\left(\varphi_{+}^{\prime \prime} \oplus \varphi_{-}^{\prime \prime}\right), \quad \operatorname{dom}_{L}^{\mathrm{D}}=\left\{\varphi \in H^{2}(I \backslash\{0\}): \varphi( \pm L)=0,\left[\varphi^{\prime}\right](0)=-\varphi(0 \pm)\right\}, \\
& \mathrm{h}_{L}^{\mathrm{N}} \varphi=-\left(\varphi_{+}^{\prime \prime} \oplus \varphi_{-}^{\prime \prime}\right), \quad \operatorname{dom}_{L}^{\mathrm{N}}=\left\{\varphi \in H^{2}(I \backslash\{0\}): \varphi^{\prime}( \pm L)=0,\left[\varphi^{\prime}\right](0)=-\varphi(0 \pm)\right\} .
\end{aligned}
$$

As $H_{0}^{1}(I)$ and $H^{1}(I)$ are compactly embedded into $L^{2}(I)$ both operators $\mathrm{h}_{L}^{\mathrm{D}}$ and $\mathrm{h}_{L}^{\mathrm{N}}$ have compact resolvent and their spectra consist of non-decreasing sequences of eigenvalues. The understanding of the first two eigenvalues of $h_{L}^{D}$ and $\mathrm{h}_{L}^{\mathrm{N}}$, as functions of $L$, is important for our purposes. The next proposition is essentially proven in the paper [22].

Proposition 3.3. [22, Prop. 2.4, Prop. 2.5] The following statements hold:

(i) there exist $L_{\mathrm{D}}>0$ and $C_{\mathrm{D}}, C_{\mathrm{D}}^{\prime}>0$ such that for all $L \geq L_{\mathrm{D}}$

$$
-\frac{1}{4}<E_{1}\left(\mathrm{~h}_{L}^{\mathrm{D}}\right) \leq-\frac{1}{4}+C_{\mathrm{D}} e^{-C_{\mathrm{D}}^{\prime} L}
$$

(ii) there exist $L_{\mathrm{N}}>0$ and $C_{\mathrm{N}}, C_{\mathrm{N}}^{\prime}>0$ such that for all $L \geq L_{\mathrm{N}}$

$$
-\frac{1}{4}>E_{1}\left(\mathrm{~h}_{L}^{\mathrm{N}}\right) \geq-\frac{1}{4}-C_{\mathrm{N}} e^{-C_{\mathrm{N}}^{\prime} L}
$$

(iii) for all $L>0, E_{2}\left(\mathrm{~h}_{L}^{\mathrm{D}}\right), E_{2}\left(\mathrm{~h}_{L}^{\mathrm{N}}\right) \geq 0$.

Further, we recall a result about another family of one-dimensional Schrödinger operators. Let us introduce the interval $J:=(1,+\infty)$ and the Hilbert space $\left(L^{2}(J),(\cdot, \cdot)_{J}\right)$. Let $c>0$ be a positive constant and $V$ be the following potential $V(x):=x^{-2}$ on $J$. We consider the following closed, densely defined, symmetric, and semibounded sesquilinear forms

$$
\begin{array}{ll}
\mathfrak{q}_{c}^{\mathrm{D}}[\varphi, \psi]=\left(\varphi^{\prime}, \psi^{\prime}\right)_{J}-c(V \varphi, \psi)_{J}, & \operatorname{dom} \mathfrak{q}_{c}^{\mathrm{D}}:=H_{0}^{1}(J), \\
\mathfrak{q}_{c}^{\mathrm{N}}[\varphi, \psi]=\left(\varphi^{\prime}, \psi^{\prime}\right)_{J}-c(V \varphi, \psi)_{J}, & \operatorname{dom} \mathfrak{q}_{c}^{\mathrm{N}}:=H^{1}(J),
\end{array}
$$

in the Hilbert space $L^{2}(J)$.

By a compact perturbation argument one can show that $\sigma_{\text {ess }}\left(\mathfrak{q}_{c}^{\mathrm{D}}\right)=\sigma_{\text {ess }}\left(\mathfrak{q}_{c}^{\mathrm{N}}\right)=[0,+\infty)$. Below we provide a result on spectral asymptotics of $\mathfrak{q}_{c}^{\mathrm{N}}$ and $\mathfrak{q}_{c}^{\mathrm{D}}$, essentially proven in [26] ( $c f$. also [24] for further generalisations).

Theorem 3.4. [26, Thm. 1] Let $c>\frac{1}{4}$. Then it holds that

$$
\mathcal{N}_{-E}\left(\mathfrak{q}_{c}^{\mathrm{D}}\right) \sim \mathcal{N}_{-E}\left(\mathfrak{q}_{c}^{\mathrm{N}}\right) \sim \frac{1}{2 \pi} \sqrt{c-\frac{1}{4}}|\ln E|, \quad E \rightarrow 0+.
$$

In particular, we have $\# \sigma_{\operatorname{dis}}\left(\mathfrak{q}_{c}^{\mathrm{D}}\right)=\# \sigma_{\mathrm{dis}}\left(\mathfrak{q}_{c}^{\mathrm{N}}\right)=\infty$.

Let us briefly indicate how the above theorem can be derived from [26]. Consider the following closed, densely defined, symmetric and semi-bounded sesquilinear form

$$
\mathfrak{q}_{c}[\varphi, \psi]:=\left(\varphi^{\prime}, \psi^{\prime}\right)_{\mathbb{R}}-c \int_{\mathbb{R}} \frac{\varphi(x) \overline{\psi(x)}}{(1+|x|)^{2}} \mathrm{~d} x, \quad \operatorname{dom} \mathfrak{q}_{c}:=H^{1}(\mathbb{R}) .
$$

By a compact perturbation argument one can show that $\sigma_{\text {ess }}\left(\mathfrak{q}_{c}\right)=[0,+\infty)$. In [26, Thm. 1] it is proven that

$$
\mathcal{N}_{-E}\left(\mathfrak{q}_{c}\right) \sim \frac{1}{\pi} \sqrt{c-\frac{1}{4}}|\ln E|, \quad E \rightarrow 0+.
$$

The self-adjoint operators on $L^{2}(\mathbb{R})$ corresponding to the forms $\mathfrak{q}_{c}^{\mathrm{N}} \oplus \mathfrak{q}_{c}^{\mathrm{N}}, \mathfrak{q}_{c}^{\mathrm{D}} \oplus \mathfrak{q}_{c}^{\mathrm{D}}$ and $\mathfrak{q}_{c}$ can be identified with self-adjoint extensions of the closed, densely defined symmetric operator on $L^{2}(\mathbb{R})$ defined as

$$
(S \varphi)(x):=-\varphi^{\prime \prime}(x)+\frac{\varphi(x)}{(1+|x|)^{2}}, \quad \operatorname{dom} S:=\left\{\varphi \in H^{2}(\mathbb{R}): \varphi(0)=\varphi^{\prime}(0)=0\right\} .
$$


As it is well known, the operator $S$ has deficiency indices $(2,2)$. Therefore, by [32, Satz 10.18] we have

$$
\left|2 \mathcal{N}_{-E}\left(\mathfrak{q}_{c}^{\mathrm{D}}\right)-\mathcal{N}_{-E}\left(\mathfrak{q}_{c}\right)\right| \leq 2 \quad \text { and } \quad\left|2 \mathcal{N}_{-E}\left(\mathfrak{q}_{c}^{\mathrm{N}}\right)-\mathcal{N}_{-E}\left(\mathfrak{q}_{c}\right)\right| \leq 2
$$

and the statement of Theorem 3.4 follows.

3.3. A lower bound on the counting function of $\mathrm{H}_{\mathcal{C}}$. In this subsection we prove Proposition 3.1. Let us briefly outline the strategy of this proof. First, we point out that the inequality in Proposition 3.1 for the counting function of $\mathrm{H}_{\mathcal{C}}$ is equivalent to the same inequality for the counting function of the form $Q_{\Gamma}$ defined in (2.18). Then, the most important part of the proof relies on a Dirichlet bracketing argument. From a geometrical point of view we construct an infinite half-strip covering a part of the meridian ray $\Gamma$, as shown in Figure 3.1. Imposing Dirichlet boundary condition on the boundary of this half-strip we obtain a new quadratic form which, in the sense of quadratic forms, is larger than $Q_{\Gamma}$. Therefore, the counting function of $Q_{\Gamma}$ can be estimated from below by the counting function of this new form. Then, we use spectral properties of the one-dimensional operators introduced in Subsection 3.2. The key point of the whole construction is that the geometry of the bracketing depends on the spectral parameter. Loosely speaking, as the spectral parameter approaches the threshold of the essential spectrum, the half-strip slides along $\Gamma$ and its width tends to infinity. In a sense, for each fixed value of the spectral parameter we employ different comparison operator to estimate the counting function of $Q_{\Gamma}$.

Proof of Proposition 3.1. Thanks to Corollary 2.7 (i) and unitary equivalence of the forms $Q_{\Gamma_{\theta}}$ (in (2.13)) and $Q_{\Gamma}$ (in (2.18)) it is sufficient to prove

$$
\liminf _{E \rightarrow 0+} \frac{\mathcal{N}_{-1 / 4-E}\left(Q_{\Gamma}\right)}{|\ln E|} \geq \frac{\cot \theta}{4 \pi} .
$$

We split the proof of this inequality into three steps.

Step 1. Let $R>0$, we define the intervals $I_{1}:=\left((\sin \theta)^{-1}+R,+\infty\right), I_{2}:=(-R \tan \theta, R \tan \theta)$, and the half-strip

$$
\Pi:=\left\{(s, t) \in \Omega_{\theta}: s>(\sin \theta)^{-1}+R,|t|<R \tan \theta\right\}=I_{1} \times I_{2} \subset \Omega_{\theta} ;
$$

where the $(s, t)$-variables and $\Omega_{\theta}$ are related to the physical domain through the change of variables (2.14) (cf. Figure 3.1). We introduce the quadratic form $Q_{\Gamma}^{\Pi}$, defined as

$$
\begin{aligned}
Q_{\Gamma}^{\Pi}[u] & :=\int_{\Pi}\left(\left|\partial_{s} u\right|^{2}+\left|\partial_{t} u\right|^{2}\right)(s \sin \theta+t \cos \theta) \mathrm{d} s \mathrm{~d} t-\int_{(\sin \theta)^{-1}+R}^{\infty}|u(s, 0)|^{2} s \sin \theta \mathrm{d} s, \\
\operatorname{dom} Q_{\Gamma}^{\Pi}: & =\left\{\left.u\right|_{\Pi}: u \in \operatorname{dom} Q_{\Gamma}, u=0 \text { on } \Omega_{\theta} \backslash \bar{\Pi}\right\} .
\end{aligned}
$$

Any $u \in \operatorname{dom} Q_{\Gamma}^{\Pi}$ can be extended by zero, defining $u_{0} \in \operatorname{dom} Q_{\Gamma}$ such that $Q_{\Gamma}^{\Pi}[u]=Q_{\Gamma}\left[u_{0}\right]$. Then, the min-max principle yields

$$
\mathcal{N}_{-1 / 4-E}\left(Q_{\Gamma}^{\Pi}\right) \leq \mathcal{N}_{-1 / 4-E}\left(Q_{\Gamma}\right)
$$

Step 2. Let us define the unitary transform

$$
\mathrm{U}: L^{2}(\Pi ;(s \sin \theta+t \cos \theta) \mathrm{d} s \mathrm{~d} t) \rightarrow L^{2}(\Pi), \quad(\mathrm{U} u)(s, t):=\sqrt{s \sin \theta+t \cos \theta} u(s, t) .
$$

By straightforward computation, the form $Q_{\Gamma}^{\Pi}$ is unitarily equivalent, via $\mathrm{U}$, to the form

$$
\begin{aligned}
\widetilde{Q}_{\Gamma}^{\Pi}[u] & :=\int_{\Pi}\left(\left|\partial_{s} u\right|^{2}+\left|\partial_{t} u\right|^{2}-\frac{1}{4(s \sin \theta+t \cos \theta)^{2}}|u|^{2}\right) \mathrm{d} s \mathrm{~d} t-\int_{(\sin \theta)^{-1}+R}^{\infty}|u(s, 0)|^{2} \mathrm{~d} s, \\
\operatorname{dom} \widetilde{Q}_{\Gamma}^{\Pi}: & =H_{0}^{1}(\Pi) .
\end{aligned}
$$

Next, we bound $(s \sin \theta+t \cos \theta)^{2}$ from above by $\sin ^{2} \theta(s+R)^{2}$, obtaining

$$
\widetilde{Q}_{\Gamma}^{\Pi}[u] \leq \int_{\Pi}\left(\left|\partial_{s} u\right|^{2}+\left|\partial_{t} u\right|^{2}-\frac{1}{4 \sin ^{2} \theta(s+R)^{2}}|u|^{2}\right) \mathrm{d} s \mathrm{~d} t-\int_{(\sin \theta)^{-1}+R}^{\infty}|u(s, 0)|^{2} \mathrm{~d} s .
$$




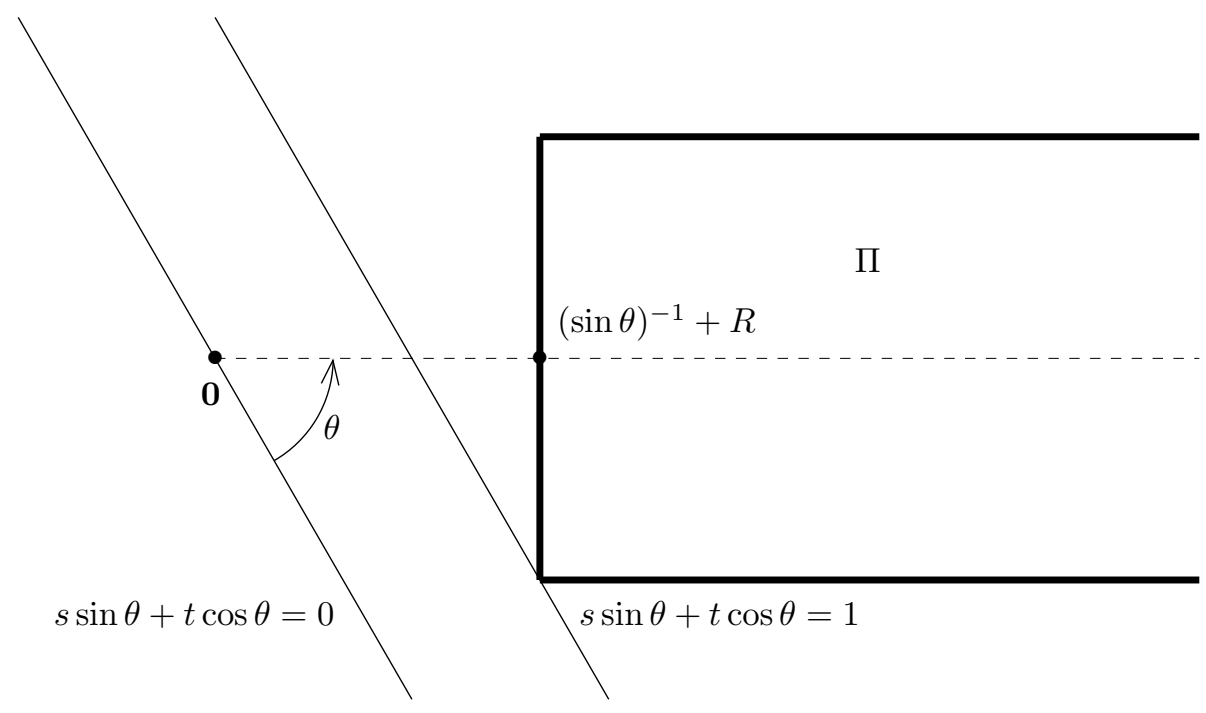

FIGURE 3.1. The inclined half-plane $\Omega_{\theta}$ and the half-strip $\Pi$. The dashed line is the meridian ray $\Gamma$.

The right hand side of (3.5) has two blocks with separated variables. Since the Hilbert space $L^{2}(\Pi)$ decomposes as $L^{2}\left(I_{1}\right) \otimes L^{2}\left(I_{2}\right)$, the form on the right hand side of (3.5) admits the respective representation

$$
\mathfrak{q}_{1, R} \otimes \mathfrak{i}_{2}+\mathfrak{i}_{1} \otimes \mathfrak{q}_{2, R}
$$

where $\mathfrak{i}_{k}, k=1,2$, is the form of the identity operator on $L^{2}\left(I_{k}\right)$; the forms $\mathfrak{q}_{k, R}, k=1,2$, are defined in the Hilbert spaces $L^{2}\left(I_{k}\right), k=1,2$, as

$$
\begin{array}{ll}
\mathfrak{q}_{1, R}[\varphi, \psi]:=\left(\varphi^{\prime}, \psi^{\prime}\right)_{I_{1}}-\left(V_{R} \varphi, \psi\right)_{I_{1}}, & \operatorname{dom} \mathfrak{q}_{1, R}:=H_{0}^{1}\left(I_{1}\right), \\
\mathfrak{q}_{2, R}[\varphi, \psi]:=\left(\varphi^{\prime}, \psi^{\prime}\right)_{I_{2}}-\varphi(0) \overline{\psi(0)}, & \operatorname{dom} \mathfrak{q}_{2, R}:=H_{0}^{1}\left(I_{2}\right) ;
\end{array}
$$

where the potential $V_{R}$ is given by $V_{R}(s):=\frac{1}{4 \sin ^{2} \theta(s+R)^{2}}$. Using the unitary operator

$$
\mathrm{V}: L^{2}(1,+\infty) \rightarrow L^{2}\left(I_{1}\right), \quad(\mathrm{V} \varphi)(s):=\frac{1}{\sqrt{2 R+(\sin \theta)^{-1}}} \varphi\left(\frac{s+R}{2 R+(\sin \theta)^{-1}}\right),
$$

one finds that the forms $\mathfrak{q}_{1, R}$ and $\left(2 R+(\sin \theta)^{-1}\right)^{-2} \mathfrak{q}_{c}^{\mathrm{D}}$ with $c=1 /\left(4 \sin ^{2} \theta\right)$ are unitarily equivalent (cf. Subsection 3.2). Thanks to (3.3), (3.5), (3.6), and the min-max principle, we obtain

$$
\begin{aligned}
\mathcal{N}_{-1 / 4-E}\left(Q_{\Gamma}\right) & \geq \#\left\{(k, j) \in \mathbb{N}^{2}: E_{k}\left(\mathfrak{q}_{1, R}\right)+E_{j}\left(\mathfrak{q}_{2, R}\right) \leq-1 / 4-E\right\} \\
& =\sum_{j=1}^{\infty} \#\left\{k \in \mathbb{N}: E_{k}\left(\mathfrak{q}_{1, R}\right) \leq-1 / 4-E-E_{j}\left(\mathfrak{q}_{2, R}\right)\right\} \\
& \geq \#\left\{k \in \mathbb{N}: E_{k}\left(\mathfrak{q}_{1, R}\right) \leq-1 / 4-E-E_{1}\left(\mathfrak{q}_{2, R}\right)\right\} .
\end{aligned}
$$

This inequality yields

$$
\mathcal{N}_{-1 / 4-E}\left(Q_{\Gamma}\right) \geq \mathcal{N}_{-1 / 4-E-E_{1}\left(\mathfrak{q}_{2, R}\right)}\left(\mathfrak{q}_{1, R}\right) .
$$

Step 3. Now, we choose $R$ depending on the spectral parameter $E>0$ as follows

$$
R=R(E):=M|\ln E|, \quad M>0,
$$

in particular, we have $R(E) \rightarrow+\infty$ as $E \rightarrow 0+$. Let the constants $C_{\mathrm{D}}, C_{\mathrm{D}}^{\prime}$ and $L_{\mathrm{D}}$ be as in Proposition 3.3 (i). Next, we choose $M>0$ sufficiently large such that $C_{\mathrm{D}}^{\prime} M \tan \theta>1$. Then for $E>0$ sufficiently small such that $\ln E<0$ and $M|\ln E| \tan \theta>L_{\mathrm{D}}$, by Proposition 3.3 (i) we have

$$
\left|1 / 4+E_{1}\left(\mathfrak{q}_{2, R(E)}\right)\right| \leq C_{\mathrm{D}} \exp \left(C_{\mathrm{D}}^{\prime} M \tan \theta \ln E\right)=C_{\mathrm{D}} E^{C_{\mathrm{D}}^{\prime} M \tan \theta}=o(E), \quad E \rightarrow 0+.
$$


Hence,

(3.9) $f(E):=\left(1 / 4+E+E_{1}\left(\mathfrak{q}_{2, R(E)}\right)\right)\left(2 R(E)+(\sin \theta)^{-1}\right)^{2}=4 M^{2} E|\ln E|^{2}+o\left(E|\ln E|^{2}\right), \quad E \rightarrow 0+$.

Using (3.8), unitary equivalence of $\mathfrak{q}_{1, R}$ and $\left(2 R+(\sin \theta)^{-1}\right)^{-2} \mathfrak{q}_{c}^{\mathrm{D}}$ and Theorem 3.4 we get

$$
\begin{aligned}
\liminf _{E \rightarrow 0+} \frac{\mathcal{N}_{-1 / 4-E}\left(Q_{\Gamma}\right)}{|\ln (E)|} & \geq \liminf _{E \rightarrow 0+} \frac{\mathcal{N}_{-1 / 4-E-E_{1}\left(\mathfrak{q}_{2, R(E)}\right)\left(\mathfrak{q}_{1, R(E)}\right)}^{|\ln (E)|}}{}=\liminf _{E \rightarrow 0+} \frac{\mathcal{N}_{-f(E)}\left(\mathfrak{q}_{c}^{\mathrm{D}}\right)}{|\ln (E)|}=\frac{\cot \theta}{4 \pi} \liminf _{E \rightarrow 0+} \frac{|\ln f(E)|}{|\ln E|}=\frac{\cot \theta}{4 \pi},
\end{aligned}
$$

where we used that $\frac{|\ln f(E)|}{|\ln E|} \rightarrow 1$ as $E \rightarrow 0+(c f$. (3.9)). It ends the proof of Proposition 3.1.

3.4. An upper bound on the counting function of $\mathrm{H}_{\mathcal{C}}$. The aim of this subsection is to prove Proposition 3.2. First, we provide an auxiliary lemma, whose proof is postponed to the end of the subsection. To formulate this lemma, for $K>0$, we define the domain

$$
\Omega_{\theta}^{K}:=\left\{(s, t) \in \mathbb{R}^{2}: s \sin \theta+t \cos \theta>2 K\right\} \subset \Omega_{\theta},
$$

and introduce the following symmetric quadratic form on the Hilbert space $L^{2}\left(\Omega_{\theta}^{K}\right)$

$$
\begin{aligned}
Q_{\Omega_{\theta}^{K}}[u] & :=\int_{\Omega_{\theta}^{K}}\left|\partial_{s} u\right|^{2}+\left|\partial_{t} u\right|^{2}-\frac{|u|^{2}}{4(s \sin \theta+t \cos \theta)^{2}} \mathrm{~d} s \mathrm{~d} t-\int_{2 K(\sin \theta)^{-1}}^{\infty}|u(s, 0)|^{2} \mathrm{~d} s, \\
\operatorname{dom} Q_{\Omega_{\theta}^{K}} & :=H^{1}\left(\Omega_{\theta}^{K}\right) .
\end{aligned}
$$

One can check that the form $Q_{\Omega_{\theta}^{K}}$ is closed, densely defined and semibounded in $L^{2}\left(\Omega_{\theta}^{K}\right)$.

Lemma 3.5. Let $\theta \in(0, \pi / 2)$. For all $K>0$ sufficiently large, the counting functions of $\mathrm{H}_{\mathcal{C}}$ and $Q_{\Omega_{\theta}^{K}}$ satisfy

$$
\limsup _{E \rightarrow 0+} \frac{\mathcal{N}_{-1 / 4-E}\left(\mathrm{H}_{\mathcal{C}}\right)}{|\ln E|} \leq \limsup _{E \rightarrow 0+} \frac{\mathcal{N}_{-1 / 4-E}\left(Q_{\Omega_{\theta}^{K}}\right)}{|\ln E|} .
$$

Now we have all the tools to prove Proposition 3.2. Let us briefly outline the strategy of this proof. In view of Lemma 3.5 it is enough to get the bound of Proposition 3.2 for the form $Q_{\Omega_{\theta}^{K}}$. Then, the most important part of the proof relies on a Neumann bracketing argument. As shown in Figure 3.2, we construct a finite number of boxes and an infinite half-strip covering the whole ray $\Gamma \cap \Omega_{\theta}^{K}$. Imposing Neumann boundary conditions on the boundaries of the boxes and of the half-strip we obtain a new quadratic form which, in the sense of quadratic forms, is smaller than $Q_{\Omega_{\theta}^{K}}$. Therefore, the counting function of $Q_{\Omega_{\theta}^{K}}$ can be estimated from above by the counting function of this new form which equals the sum of the counting functions corresponding to each subset of the covering. We estimate them using spectral properties of the one-dimensional operators introduced in Subsection 3.2. In this construction not only the sizes of the subsets in the covering depend on the spectral parameter, but also their number. Loosely speaking, as the spectral parameter approaches the threshold of the essential spectrum, the half-strip slides along the meridian ray and its width tends to infinity; the size of each box varies while their number tends to infinity.

Proof of Proposition 3.2. According to Lemma 3.5 it is sufficient to prove that for a fixed $K>0$ sufficiently large, we have the bound

$$
\limsup _{E \rightarrow 0+} \frac{\mathcal{N}_{-1 / 4-E}\left(Q_{\Omega_{\theta}^{K}}\right)}{|\ln E|} \leq \frac{\cot \theta}{4 \pi}
$$

As in the proof of Proposition 3.1, we split the proof of this inequality into three steps.

Step 1. Let us introduce the parameters: $R>0, m:=\lfloor\sqrt{R}\rfloor, r:=2 K(\sin \theta)^{-1}$ and the sequences $r_{k}:=3 r+k R / m$, $d_{k}:=\left(r_{k} \tan \theta\right) / 2($ for $k=0,1,2, \ldots, m)$. For the sake of convenience we set $r_{m+1}=+\infty$. We introduce the domains

$$
\Lambda_{k}:=\left\{(s, t) \in \mathbb{R}^{2}: s \in\left(r_{k}, r_{k+1}\right), t \in\left(-d_{k}, d_{k}\right)\right\}, \quad k=0,1, \ldots, m .
$$


The inclusions $\Lambda_{k} \subset \Omega_{\theta}^{K}$ hold for all $k=0,1, \ldots, m$. Indeed, for any $(s, t) \in \Lambda_{k}$ we have

$$
s \sin \theta+t \cos \theta>r_{k} \sin \theta-d_{k} \cos \theta=\frac{r_{k} \sin \theta}{2} \geq \frac{3 r \sin \theta}{2}=\frac{6 K}{2}=3 K>2 K .
$$

We also define the domains $\Lambda_{m+1}, \Lambda_{m+2} \subset \Omega_{\theta}^{K}(c f$. Figure 3.2) as

$$
\Lambda_{m+1}:=\left\{(s, t) \in \Omega_{\theta}^{K}: s<6 K(\sin \theta)^{-1},|t|<K(\cos \theta)^{-1}\right\}, \quad \Lambda_{m+2}:=\Omega_{\theta}^{K} \backslash \overline{\cup_{k=0}^{m+1} \Lambda_{k}} .
$$
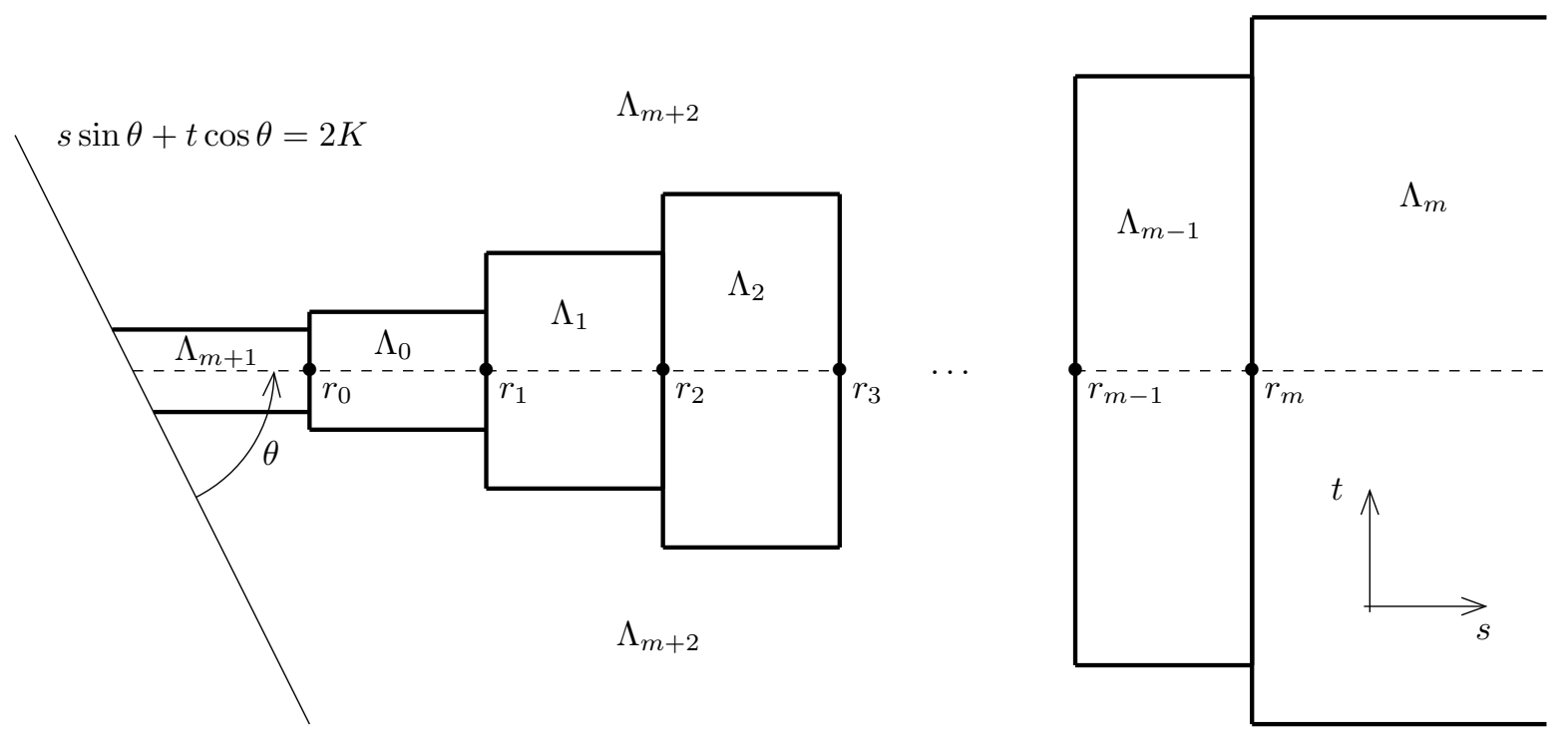

FIGURE 3.2. Sketch of the different subdomains $\Lambda_{k}$ of $\Omega_{\theta}^{K}(k \in\{0, \ldots, m+2\})$. The dashed line is the meridian ray $\Gamma \cap \Omega_{\theta}^{K}$.

We introduce the notation $u_{k}=\left.u\right|_{\Lambda_{k}}(k=0,1, \ldots,(m+2))$ and we consider the following closed, densely defined, symmetric and semibounded quadratic form $\widetilde{Q}_{\Omega_{\theta}^{K}}$ in $L^{2}\left(\Omega_{\theta}^{K}\right)$, defined as

$$
\begin{aligned}
\widetilde{Q}_{\Omega_{\theta}^{K}}[u] & :=\sum_{k=0}^{m+2}\left\|\nabla u_{k}\right\|_{\Lambda_{k}}^{2}-\int_{\Omega_{\theta}^{K}} \frac{|u|^{2}}{4(s \sin \theta+t \cos \theta)^{2}} \mathrm{~d} s \mathrm{~d} t-\int_{2 K(\sin \theta)^{-1}}^{\infty}|u(s, 0)|^{2} \mathrm{~d} s, \\
\operatorname{dom} \widetilde{Q}_{\Omega_{\theta}^{K}} & :=\bigoplus_{k=0}^{m+2} H^{1}\left(\Lambda_{k}\right) .
\end{aligned}
$$

This form admits a natural decomposition into parts corresponding to the sub-domains $\Lambda_{k}$

$$
\widetilde{Q}_{\Omega_{\theta}^{K}}[u]=\sum_{k=0}^{m+2} Q_{\Lambda_{k}}\left[u_{k}\right],
$$

where, for $k=0,1, \ldots,(m+2)$, the quadratic forms $Q_{\Lambda_{k}}$ have domains $\operatorname{dom} Q_{\Lambda_{k}}:=H^{1}\left(\Lambda_{k}\right)$ and are given by

$$
\begin{aligned}
Q_{\Lambda_{k}}[u] & :=\|\nabla u\|_{\Lambda_{k}}^{2}-\int_{\Lambda_{k}} \frac{|u|^{2}}{4(s \sin \theta+t \cos \theta)^{2}} \mathrm{~d} s \mathrm{~d} t-\int_{r_{k}}^{r_{k+1}}|u(s, 0)|^{2} \mathrm{~d} s, \quad k=0, \ldots m \\
Q_{\Lambda_{m+1}}[u] & :=\|\nabla u\|_{\Lambda_{m+1}}^{2}-\int_{\Lambda_{m+1}} \frac{|u|^{2}}{4(s \sin \theta+t \cos \theta)^{2}} \mathrm{~d} s \mathrm{~d} t-\int_{2 K(\sin \theta)^{-1}}^{r_{0}}|u(s, 0)|^{2} \mathrm{~d} s, \\
Q_{\Lambda_{m+2}}[u] & :=\|\nabla u\|_{\Lambda_{m+2}}^{2}-\int_{\Lambda_{m+2}} \frac{|u|^{2}}{4(s \sin \theta+t \cos \theta)^{2}} \mathrm{~d} s \mathrm{~d} t .
\end{aligned}
$$


As $\operatorname{dom} Q_{\Omega_{\theta}^{K}} \subset \operatorname{dom} \widetilde{Q}_{\Omega_{\theta}^{K}}$, for any $u \in \operatorname{dom} Q_{\Omega_{\theta}^{K}}$ we have $Q_{\Omega_{\theta}^{K}}[u]=\widetilde{Q}_{\Omega_{\theta}^{K}}[u]$ and we get the form ordering $\widetilde{Q}_{\Omega_{\theta}^{K}} \prec Q_{\Omega_{\theta}^{K}}$. The min-max principle yields, for all $E>0$, the bound

$$
\mathcal{N}_{-1 / 4-E}\left(Q_{\Omega_{\theta}^{K}}\right) \leq \sum_{k=0}^{m+2} \mathcal{N}_{-1 / 4-E}\left(Q_{\Lambda_{k}}\right) .
$$

Step 2. In this step we obtain bounds on the functions $E \mapsto \mathcal{N}_{-1 / 4-E}\left(Q_{\Lambda_{k}}\right)(k=0,1,2, \ldots,(m+2))$. First, we bound from above the functions

$$
E \mapsto \mathcal{N}_{-1 / 4-E}\left(Q_{\Lambda_{m+1}}\right) \quad \text { and } \quad E \mapsto \mathcal{N}_{-1 / 4-E}\left(Q_{\Lambda_{m+2}}\right) .
$$

Because $H^{1}\left(\Lambda_{m+1}\right)$ is compactly embedded into $L^{2}\left(\Lambda_{m+1}\right)$ the quadratic form $Q_{\Lambda_{m+1}}$ is associated with an operator with compact resolvent. Therefore, since the domain $\Lambda_{m+1}$ does not depend on $R$, there exists a constant $\mathfrak{c}_{\theta}=\mathfrak{c}_{\theta}(K)>0$, which depends on $\theta$ and $K$ (but not on $R$ ), such that, for any $E>0$

$$
\mathcal{N}_{-1 / 4-E}\left(Q_{\Lambda_{m+1}}\right) \leq \mathcal{N}_{-1 / 4}\left(Q_{\Lambda_{m+1}}\right)=\mathfrak{c}_{\theta} .
$$

Further, for any $u \in \operatorname{dom} Q_{\Lambda_{m+2}}$, we have

$$
Q_{\Lambda_{m+2}}[u] \geq\|\nabla u\|_{\Lambda_{m+2}}^{2}-\frac{1}{16 K^{2}}\|u\|_{\Lambda_{m+2}}^{2} .
$$

Consequently, for a fixed $K>0$ such that $\frac{1}{16 K^{2}}<1 / 4$, the min-max principle yields $\mathcal{N}_{-1 / 4}\left(Q_{\Lambda_{m+2}}\right)=0$. Hence, for any $E>0$, the following equation holds

$$
\mathcal{N}_{-1 / 4-E}\left(Q_{\Lambda_{m+2}}\right) \leq \mathcal{N}_{-1 / 4}\left(Q_{\Lambda_{m+2}}\right)=0
$$

Next, we obtain upper bounds for the functions

$$
E \mapsto \sum_{k=0}^{m-1} \mathcal{N}_{-1 / 4-E}\left(Q_{\Lambda_{k}}\right) \quad \text { and } \quad E \mapsto \mathcal{N}_{-1 / 4-E}\left(Q_{\Lambda_{m}}\right)
$$

To this end we define the potential $V_{m}(s)=\frac{1}{4 \sin ^{2} \theta\left(s-r_{m} / 2\right)^{2}}$ and the non-increasing sequence

$$
\varepsilon_{k}:=\sup _{(s, t) \in \Lambda_{k}} \frac{1}{4(s \sin \theta+t \cos \theta)^{2}}=\frac{1}{r_{k}^{2} \sin ^{2} \theta}, \quad k=0,1, \ldots,(m-1) .
$$

We observe that for any $u \in \operatorname{dom} Q_{\Lambda_{k}}$

$$
\begin{aligned}
& Q_{\Lambda_{k}}[u] \geq\|\nabla u\|_{\Lambda_{k}}^{2}-\varepsilon_{k}\|u\|_{\Lambda_{k}}^{2}-\int_{r_{k}}^{r_{k+1}}|u(s, 0)|^{2} \mathrm{~d} s, \quad k=0,1, \ldots,(m-1), \\
& Q_{\Lambda_{m}}[u] \geq\|\nabla u\|_{\Lambda_{m}}^{2}-\left(V_{m} u, u\right)_{\Lambda_{m}}-\int_{r_{m}}^{r_{m+1}}|u(s, 0)|^{2} \mathrm{~d} s .
\end{aligned}
$$

Then, we define the intervals $I_{1}^{k}=\left(r_{k}, r_{k+1}\right), I_{2}^{k}=\left(-d_{k}, d_{k}\right)$, and the following symmetric sesquilinear forms

$$
\begin{array}{lll}
\mathfrak{q}_{1, R}^{k}[\varphi, \psi]:=\left(\varphi^{\prime}, \psi^{\prime}\right)_{I_{1}^{k}}-\varepsilon_{k}(\varphi, \psi)_{I_{1}^{k}}, & \operatorname{dom} \mathfrak{q}_{1, R}^{k}:=H^{1}\left(I_{1}^{k}\right), & k=0,1, \ldots,(m-1), \\
\mathfrak{q}_{1, R}^{m}[\varphi, \psi]:=\left(\varphi^{\prime}, \psi^{\prime}\right)_{1}^{m}-\left(V_{m} \varphi, \psi\right)_{I_{1}^{m}}, & \operatorname{dom} \mathfrak{q}_{1, R}^{m}:=H^{1}\left(I_{1}^{m}\right), & \\
\mathfrak{q}_{2, R}^{k}[\varphi, \psi]:=\left(\varphi^{\prime}, \psi^{\prime}\right)_{I_{2}^{k}}-\varphi(0) \overline{\psi(0)}, & \operatorname{dom} \mathfrak{q}_{2, R}^{k}:=H^{1}\left(I_{2}^{k}\right), \quad k=0,1, \ldots, m .
\end{array}
$$

One can check that all the forms are closed, densely defined and semibounded in $L^{2}$-spaces over their respective intervals. As $L^{2}\left(\Lambda_{k}\right)=L^{2}\left(I_{1}^{k}\right) \otimes L^{2}\left(I_{2}^{k}\right)$, we introduce the quadratic forms

$$
\widetilde{Q}_{\Lambda_{k}}:=\mathfrak{q}_{1, R}^{k} \otimes \mathfrak{i}_{2}^{k}+\mathfrak{i}_{1}^{k} \otimes \mathfrak{q}_{2, R}^{k}, \quad k=0,1, \ldots, m,
$$


and the form orderings $\widetilde{Q}_{\Lambda_{k}} \prec Q_{\Lambda_{k}}$ hold for all $k=0,1, \ldots, m$. Here, for $j=1,2$ and $k=0,2, \ldots, m, \mathfrak{i}_{j}^{k}$ denote the forms of the identity operators in $L^{2}\left(I_{j}^{k}\right)$. Hence, we arrive at the bound

$$
\begin{aligned}
\mathcal{N}_{-1 / 4-E}\left(Q_{\Lambda_{k}}\right) & \leq \mathcal{N}_{-1 / 4-E}\left(\widetilde{Q}_{\Lambda_{k}}\right)=\#\left\{(l, j) \in \mathbb{N}^{2}: E_{l}\left(\mathfrak{q}_{1, R}^{k}\right)+E_{j}\left(\mathfrak{q}_{2, R}^{k}\right) \leq-1 / 4-E\right\} \\
& =\sum_{j=1}^{\infty} \#\left\{l \in \mathbb{N}: E_{l}\left(\mathfrak{q}_{1, R}^{k}\right) \leq-1 / 4-E-E_{j}\left(\mathfrak{q}_{2, R}^{k}\right)\right\} .
\end{aligned}
$$

Now, we choose $K>0$ sufficiently large such that $\varepsilon_{1}<1 / 4$. Thanks to Proposition 3.3 (iii), we know that all the summands, for $j>1$, in the above sum equal to zero. Thus, we get the same bound in a simplified form

$$
\mathcal{N}_{-1 / 4-E}\left(Q_{\Lambda_{k}}\right) \leq \#\left\{l \in \mathbb{N}: E_{l}\left(\mathfrak{q}_{1, R}^{k}\right) \leq-1 / 4-E-E_{1}\left(\mathfrak{q}_{2, R}^{k}\right)\right\}
$$

For $k=0,1, \ldots,(m-1)$ we deduce from (3.15) using (3.14) and Proposition 3.3 (ii) that for $R>0$ sufficiently large

$$
\begin{aligned}
\mathcal{N}_{-1 / 4-E}\left(Q_{\Lambda_{k}}\right) & \leq \#\left\{l \in \mathbb{N}_{0}: m^{2} \pi^{2} l^{2} R^{-2} \leq-1 / 4+\varepsilon_{k}-E_{1}\left(\mathfrak{q}_{2, R}^{k}\right)\right\} \\
& \leq 1+C_{1} \sqrt{R} \sqrt{e^{-C_{2} r_{k}}+r_{k}^{-2}} \leq 1+\frac{C_{3} \sqrt{R}}{r_{k}}
\end{aligned}
$$

where the positive constants $C_{1}, C_{2}$ and $C_{3}$ do not depend on $R$. Summing the estimates (3.16) over $k$, we end up with

$$
\begin{aligned}
\sum_{k=0}^{m-1} \mathcal{N}_{-1 / 4-E}\left(Q_{\Lambda_{k}}\right) & \leq m+C_{3} \sqrt{R} \sum_{k=0}^{m-1} \frac{1}{r_{k}} \\
& \leq m+C_{3} \sqrt{R} \frac{1}{r_{0}}+C_{3} \int_{0}^{R} \frac{\mathrm{d} x}{3 r+x} \leq C_{4} \sqrt{R}
\end{aligned}
$$

for all $R>0$ sufficiently large and a positive constant $C_{4}$ which does not depend on $R$.

Further, for $k=m$ we obtain from (3.15)

$$
\begin{aligned}
\mathcal{N}_{-1 / 4-E}\left(Q_{\Lambda_{m}}\right) & \leq \#\left\{l \in \mathbb{N}: E_{l}\left(\mathfrak{q}_{1, R}^{m}\right) \leq-1 / 4-E-E_{1}\left(\mathfrak{q}_{2, R}^{m}\right)\right\} \\
& \leq \mathcal{N}_{-1 / 4-E-E_{1}\left(\mathfrak{q}_{2, R}^{m}\right)}\left(\mathfrak{q}_{1, R}^{m}\right) .
\end{aligned}
$$

Using the unitary operator

$$
\mathrm{U}: L^{2}(1,+\infty) \rightarrow L^{2}\left(I_{1}^{m}\right), \quad(\mathrm{U} \psi)(s):=\sqrt{\frac{2}{r_{m}}} \psi\left(\frac{2 s}{r_{m}}-1\right),
$$

one finds by direct computations that the forms $\mathfrak{q}_{1, R}^{m}$ and $\frac{4}{r_{m}^{2}} \mathfrak{q}_{c}^{\mathrm{N}}$ with $c=1 /\left(4 \sin ^{2} \theta\right)$ are unitarily equivalent; where the form $\mathfrak{q}_{c}^{\mathrm{N}}$ is defined in (3.2).

Combining the bound (3.11) and the estimates (3.12), (3.13), (3.17), (3.18) we obtain

$$
\mathcal{N}_{-1 / 4-E}\left(Q_{\Omega_{\theta}^{K}}\right) \leq \mathfrak{c}_{\theta}+C_{4} \sqrt{R}+\mathcal{N}_{-1 / 4-E-E_{1}\left(\mathfrak{q}_{2, R}^{m}\right)}\left(\mathfrak{q}_{1, R}^{m}\right)
$$

Step 3. Now, we choose $R$ depending on the spectral parameter $E>0$ as follows

$$
R=R(E):=M|\ln E|, \quad M>0,
$$

in particular, we have $R(E) \rightarrow+\infty$ as $E \rightarrow 0+$. Let the constants $C_{\mathrm{N}}, C_{\mathrm{N}}^{\prime}$ and $L_{\mathrm{N}}$ be as in Proposition 3.3 (ii). Next, we choose $M>0$ sufficiently large such that $C_{\mathrm{N}}^{\prime} M \tan \theta>2$. Then, for $E>0$ sufficiently small such that $\ln E<0$ and $M|\ln E| \tan \theta / 2>L_{\mathrm{N}}$, by Proposition 3.3 (ii) we have

$$
\left|1 / 4+E_{1}\left(\mathfrak{q}_{2, R(E)}^{m}\right)\right| \leq C_{\mathrm{N}} \exp \left(\left(C_{\mathrm{N}}^{\prime} \tan \theta / 2\right)(3 r+M|\ln E|)\right)=\widetilde{C}_{\mathrm{N}} E^{\left(C_{\mathrm{N}}^{\prime} M \tan \theta\right) / 2}=o(E), \quad E \rightarrow 0+,
$$

where $\widetilde{C}_{\mathrm{N}}>0$. Hence,

$$
f(E):=\left(r_{m}(E)^{2} / 4\right)\left(1 / 4+E+E_{1}\left(\mathfrak{q}_{2, R(E)}\right)\right)=M^{2} E|\ln E|^{2} / 4+o\left(E|\ln E|^{2}\right), \quad E \rightarrow 0+.
$$


Using (3.8), unitary equivalence of $\mathfrak{q}_{1, R}^{m}$ and $\frac{4}{r_{m}^{2}} \mathfrak{q}_{c}^{\mathrm{N}}$ and Theorem 3.4 we get

$$
\begin{aligned}
\limsup _{E \rightarrow 0+} \frac{\mathcal{N}_{-1 / 4-E}\left(Q_{\Omega_{\theta}^{K}}\right)}{|\ln E|} & \leq \limsup _{E \rightarrow 0+}\left(\frac{\mathfrak{c}_{\theta}}{|\ln E|}+\frac{C_{4} \sqrt{M}}{\sqrt{|\ln E|}}+\frac{\mathcal{N}_{-1 / 4-E-E_{1}\left(\mathfrak{q}_{2, R(E)}^{m}\right)}\left(\mathfrak{q}_{1, R(E)}^{m}\right)}{|\ln E|}\right) \\
& \leq \limsup _{E \rightarrow 0+} \frac{\mathcal{N}_{-f(E)}\left(\mathfrak{q}_{c}^{\mathrm{N}}\right)}{|\ln E|}=\frac{\cot \theta}{4 \pi} \limsup _{E \rightarrow 0+} \frac{|\ln f(E)|}{|\ln E|}=\frac{\cot \theta}{4 \pi} .
\end{aligned}
$$

where we used that $\frac{|\ln f(E)|}{|\ln E|} \rightarrow 1$ as $E \rightarrow 0+(c f$. (3.20)). This ends the proof of Proposition 3.2.

Now we provide the proof of Lemma 3.5.

Proof of Lemma 3.5. Thanks to Corollary 2.7 (i) it is sufficient to prove that

$$
\limsup _{E \rightarrow 0+} \frac{\mathcal{N}_{-1 / 4-E}\left(Q_{\Gamma_{\theta}}\right)}{|\ln E|} \leq \limsup _{E \rightarrow 0+} \frac{\mathcal{N}_{-1 / 4-E}\left(Q_{\Omega_{\theta}^{K}}\right)}{|\ln E|},
$$

where the forms $Q_{\Gamma_{\theta}}$ and $Q_{\Omega_{\theta}^{K}}$ are defined as in (2.13) and in (3.10), respectively.

Step 1. Using an IMS formula we split the quadratic form $Q_{\Gamma_{\theta}}$ into two forms, one acting on a strip-shaped geometrical domain attached to the boundary $\partial \mathbb{R}_{+}^{2}$ of $\mathbb{R}_{+}^{2}$, the other one acting away from it. For this purpose, let us introduce a $\mathcal{C}^{\infty}$-smooth cut-off function $\chi_{0}: \mathbb{R}_{+} \rightarrow[0,1]$ such that

$$
\chi_{0}(r):= \begin{cases}1, & r \leq 1, \\ 0, & r \geq 2 .\end{cases}
$$

We also introduce the function $\chi_{1}: \mathbb{R}_{+} \rightarrow[0,1]$ such that $\chi_{0}^{2}+\chi_{1}^{2} \equiv 1$. Now, for $K>0$, we define $\chi_{j}^{K}(r):=$ $\chi_{j}\left(K^{-1} r\right), j=0,1$, and introduce the following bounded function

$$
W^{K}(r):=\left|\left(\chi_{0}^{K}\right)^{\prime}(r)\right|^{2}+\left|\left(\chi_{1}^{K}\right)^{\prime}(r)\right|^{2}=K^{-2}\left(\left|\chi_{0}^{\prime}\left(K^{-1} r\right)\right|^{2}+\left|\chi_{1}^{\prime}\left(K^{-1} r\right)\right|^{2}\right) .
$$

We set $W:=W^{1}$ (for $K=1$ ) and observe that $\left\|W^{K}\right\|_{\infty}=K^{-2}\|W\|_{\infty}$. Moreover, for any $u \in \operatorname{dom} Q_{\Gamma_{\theta}}$, a simple computation (cf. [10, Sec. 3.1]) yields

$$
Q_{\Gamma_{\theta}}[u]=Q_{\Gamma_{\theta}}\left[\chi_{0}^{K} u\right]+Q_{\Gamma_{\theta}}\left[\chi_{1}^{K} u\right]-\int_{\mathbb{R}_{+}^{2}} W^{K}(r)|u(r, z)|^{2} r \mathrm{~d} r \mathrm{~d} z
$$

Next we introduce the sub-domains

$$
\Omega_{0}:=\left\{(r, z) \in \mathbb{R}_{+}^{2}: r \leq 2 K\right\}, \quad \Omega_{1}:=\left\{(r, z) \in \mathbb{R}_{+}^{2}: r \geq K\right\}
$$

of the meridian domain $\mathbb{R}_{+}^{2}$ (note that $\operatorname{supp} W^{K} \subset \Omega_{0}$ ). For $j=0$, we set $\Gamma_{j}=\Gamma_{\theta} \cap \Omega_{j}$ and define $I_{0}:=$ $\left(0,2 K(\sin \theta)^{-1}\right), I_{1}:=\left(K(\sin \theta)^{-1},+\infty\right), \Sigma_{0}=\{2 K\} \times \mathbb{R}$, and $\Sigma_{1}=\{K\} \times \mathbb{R}$. Then we consider the quadratic forms $Q_{j}, j=0,1$, defined as

$$
\begin{aligned}
Q_{j}[u] & :=\int_{\Omega_{j}}\left(\left|\partial_{r} u\right|^{2}+\left|\partial_{z} u\right|^{2}-W^{K}(r)|u|^{2}\right) r \mathrm{~d} r \mathrm{~d} z-\int_{I_{j}}|u(s \sin \theta, s \cos \theta)|^{2} s \sin \theta \mathrm{d} s, \\
\operatorname{dom} Q_{j} & :=\left\{\left.u\right|_{\Omega_{j}}: u \in \operatorname{dom} Q_{\Gamma_{\theta}},\left.u\right|_{\Sigma_{j}}=0\right\} .
\end{aligned}
$$

Thanks to (3.22), we get that, for $j=0,1$ any $u \in \operatorname{dom} Q_{\Gamma_{\theta}}, \chi_{j}^{K} u \in \operatorname{dom} Q_{j}$ and we have the relation

$$
Q_{\Gamma_{\theta}}[u]=Q_{0}\left[\chi_{0}^{K} u\right]+Q_{1}\left[\chi_{1}^{K} u\right] .
$$

Using [11, Lem. 5.2] we find

$$
\mathcal{N}_{-1 / 4-E}\left(Q_{\Gamma_{\theta}}\right) \leq \mathcal{N}_{-1 / 4-E}\left(Q_{0}\right)+\mathcal{N}_{-1 / 4-E}\left(Q_{1}\right)
$$

Step 2. In this step we prove that for any $K>0$ sufficiently large, there exists a constant $\widehat{\mathfrak{c}}_{\theta}=\widehat{\mathfrak{c}}_{\theta}(K)>0$ such that

$$
\mathcal{N}_{-1 / 4-E}\left(Q_{0}\right) \leq \widehat{\mathfrak{c}}_{\theta}
$$


for all $E>0$. First, we introduce the quadratic form $Q_{0}^{\prime}$, defined as

$$
Q_{0}^{\prime}[u]:=\int_{\Omega_{0}}\left(\left|\partial_{r} u\right|^{2}+\left|\partial_{z} u\right|^{2}\right) r \mathrm{~d} r \mathrm{~d} z-\int_{I_{0}}|u(s \sin \theta, s \cos \theta)|^{2} s \sin \theta \mathrm{d} s, \quad \operatorname{dom} Q_{0}^{\prime}:=\operatorname{dom} Q_{0} .
$$

One can check that the above form is closed, symmetric, densely defined and semibounded in $L^{2}\left(\Omega_{0} ; r \mathrm{~d} r \mathrm{~d} z\right)$. Now, for any $u \in \operatorname{dom} Q_{0}$ we have

$$
Q_{0}[u] \geq Q_{0}^{\prime}[u]-K^{-2}\|W\|_{\infty}\|u\|_{L^{2}\left(\Omega_{0} ; r \mathrm{~d} r \mathrm{~d} z\right)}^{2}
$$

Consequently, we get that

$$
\mathcal{N}_{-1 / 4-E}\left(Q_{0}\right) \leq \mathcal{N}_{-1 / 4-E+K^{-2}\|W\|_{\infty}}\left(Q_{0}^{\prime}\right) .
$$

Next, we choose $K>0$ sufficiently large such that $-1 / 4+K^{-2}\|W\|_{\infty} \leq-1 / 8$. Combining (3.25) and (3.26) we obtain

$$
\mathcal{N}_{-1 / 4-E}\left(Q_{0}\right) \leq \mathcal{N}_{-1 / 8}\left(Q_{0}^{\prime}\right)
$$

Secondly, let us split the domain $\Omega_{0}$ into two disjoint sub-domains (cf. Figure 3.3)

$$
\Omega_{00}:=\left\{(r, z) \in \Omega_{0}: z \in(0,2 K \cot \theta)\right\}, \quad \Omega_{01}:=\left\{(r, z) \in \Omega_{0}: z \notin[0,2 K \cot \theta]\right\} .
$$

For $j=0,1$, we define $\Sigma_{0 j}:=\Sigma_{0} \cap \partial \Omega_{0 j}$ and we consider the quadratic forms $Q_{00}^{\prime}$ and $Q_{01}^{\prime}$ defined as

$$
\begin{aligned}
Q_{00}^{\prime}[u] & :=\int_{\Omega_{00}}\left(\left|\partial_{r} u\right|^{2}+\left|\partial_{z} u\right|^{2}\right) r \mathrm{~d} r \mathrm{~d} z-\int_{I_{0}}|u(s \sin \theta, s \cos \theta)|^{2} s \sin \theta \mathrm{d} s, \\
Q_{01}^{\prime}[u] & :=\int_{\Omega_{01}}\left(\left|\partial_{r} u\right|^{2}+\left|\partial_{z} u\right|^{2}\right) r \mathrm{~d} r \mathrm{~d} z, \\
\operatorname{dom} Q_{0 j}^{\prime} & :=\left\{u: u, \partial_{r} u, \partial_{z} u \in L^{2}\left(\Omega_{0 j}, r \mathrm{~d} r \mathrm{~d} z\right),\left.u\right|_{\Sigma_{0 j}}=0\right\}, \quad j=0,1 .
\end{aligned}
$$

One can check that the above forms are closed, symmetric, densely defined and semibounded in $L^{2}\left(\Omega_{00} ; r \mathrm{~d} r \mathrm{~d} z\right)$ and in $L^{2}\left(\Omega_{01} ; r \mathrm{~d} r \mathrm{~d} z\right)$, respectively. For $u \in \operatorname{dom} Q_{0}^{\prime}$ and $j=0,1$, we define $u_{j}=\left.u\right|_{\Omega_{0 j}}$ and get

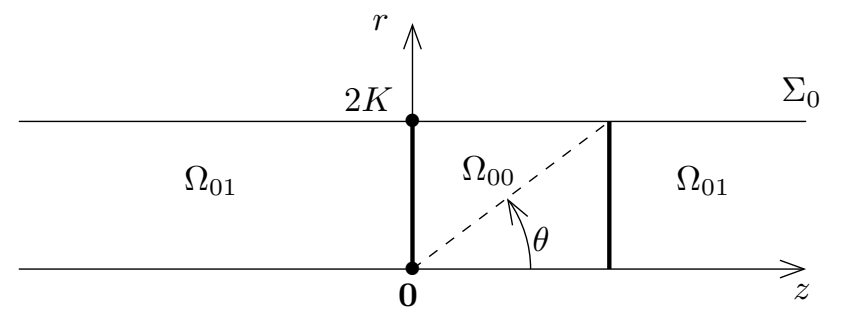

FIGURE 3.3. The domain $\Omega_{0}$ and the sub-domains $\Omega_{00}$ and $\Omega_{01}$. The dashed line is the support of the ray $\Gamma_{\theta} \cap \Omega_{0}$.

$$
Q_{0}^{\prime}[u]=Q_{00}^{\prime}\left[u_{0}\right]+Q_{01}^{\prime}\left[u_{1}\right] .
$$

The above equality and the min-max principle yield

$$
\mathcal{N}_{-1 / 8}\left(Q_{0}^{\prime}\right) \leq \mathcal{N}_{-1 / 8}\left(Q_{00}^{\prime}\right)+\mathcal{N}_{-1 / 8}\left(Q_{01}^{\prime}\right) .
$$

Note that for all $u \in \operatorname{dom} Q_{01}^{\prime}$, we have $Q_{01}^{\prime}[u] \geq 0$. Consequently, we get

$$
\mathcal{N}_{-1 / 8}\left(Q_{01}^{\prime}\right)=0 \text {. }
$$

Moreover, the quadratic form $Q_{00}^{\prime}$ is associated with the lowest fiber operator of a three-dimensional Schrödinger operator with a surface $\delta$-interaction acting on a bounded domain with mixed boundary conditions (Neumann and Dirichlet). This operator has compact resolvent and its sequence of eigenvalues goes to infinity. Hence, we obtain

$$
\mathcal{N}_{-1 / 8}\left(Q_{00}^{\prime}\right)=\widehat{\mathfrak{c}}_{\theta}<\infty,
$$

with some constant $\widehat{\mathfrak{c}}_{\theta}=\widehat{\mathfrak{c}}_{\theta}(K)>0$. Combining (3.27), (3.28), (3.29) and (3.30) we obtain (3.24). 
Step 3. We remark that the domain $\widetilde{\Omega}_{1}=\left\{(s, t) \in \mathbb{R}^{2}: s \sin \theta+t \cos \theta>K\right\} \subset \Omega_{\theta}$ is the image of $\Omega_{1}$ under rotation (2.14) and we consider the unitary transform

$$
\mathrm{U}: L^{2}\left(\Omega_{1} ; r \mathrm{~d} r \mathrm{~d} z\right) \rightarrow L^{2}\left(\widetilde{\Omega}_{1}\right), \quad(\mathrm{U} u)(s, t):=u(s \sin \theta+t \cos \theta, s \cos \theta-t \sin \theta) \sqrt{s \sin \theta+t \cos \theta} .
$$

A straightforward computation yields that the quadratic form $Q_{1}$ is unitarily equivalent, via $\mathrm{U}$, to the form

$$
\begin{aligned}
\widetilde{Q}_{1}[u] & :=\|\nabla u\|_{\widetilde{\Omega}_{1}}^{2}-\int_{\widetilde{\Omega}_{1}} \frac{|u|^{2}}{4(s \sin \theta+t \cos \theta)^{2}}+W^{K}(s \sin \theta+t \cos \theta)|u|^{2} \mathrm{~d} s \mathrm{~d} t-\int_{K(\sin \theta)^{-1}}^{\infty}|u(s, 0)|^{2} \mathrm{~d} s \\
\operatorname{dom} \widetilde{Q}_{1} & :=H_{0}^{1}\left(\widetilde{\Omega}_{1}\right) .
\end{aligned}
$$

We introduce the sub-domains of $\widetilde{\Omega}_{1}$

$$
\widetilde{\Omega}_{10}:=\left\{(s, t) \in \mathbb{R}^{2}: K<s \sin \theta+t \cos \theta<2 K\right\}, \quad \widetilde{\Omega}_{11}:=\left\{(s, t) \in \mathbb{R}^{2}: s \sin \theta+t \cos \theta>2 K\right\},
$$

and the forms

$$
\begin{aligned}
& \widetilde{Q}_{10}[u]:=\|\nabla u\|_{\widetilde{\Omega}_{10}}^{2}-\int_{\widetilde{\Omega}_{10}} \frac{|u|^{2}}{4(s \sin \theta+t \cos \theta)^{2}}+W^{K}(s \sin \theta+t \cos \theta)|u|^{2} \mathrm{~d} s \mathrm{~d} t-\int_{K(\sin \theta)^{-1}}^{2 K(\sin \theta)^{-1}}|u(s, 0)|^{2} \mathrm{~d} s, \\
& \widetilde{Q}_{11}[u]:=\|\nabla u\|_{\widetilde{\Omega}_{11}}^{2}-\int_{\widetilde{\Omega}_{11}} \frac{|u|^{2}}{4(s \sin \theta+t \cos \theta)^{2}} \mathrm{~d} s \mathrm{~d} t-\int_{2 K(\sin \theta)^{-1}}^{+\infty}|u(s, 0)|^{2} \mathrm{~d} s, \\
& \operatorname{dom} \widetilde{Q}_{10}:=\left\{u \in H^{1}\left(\widetilde{\Omega}_{10}\right):\left.u\right|_{\partial \widetilde{\Omega}_{1}}=0\right\}, \quad \operatorname{dom} \widetilde{Q}_{11}:=H^{1}\left(\widetilde{\Omega}_{11}\right) .
\end{aligned}
$$

The above forms are closed, symmetric, densely defined and semibounded in $L^{2}\left(\widetilde{\Omega}_{10}\right)$ and in $L^{2}\left(\widetilde{\Omega}_{11}\right)$, respectively. Once again, we get by the min-max principle

$$
\mathcal{N}_{-1 / 4-E}\left(Q_{1}\right)=\mathcal{N}_{-1 / 4-E}\left(\widetilde{Q}_{1}\right) \leq \mathcal{N}_{-1 / 4-E}\left(\widetilde{Q}_{10}\right)+\mathcal{N}_{-1 / 4-E}\left(\widetilde{Q}_{11}\right)
$$

Step 4. In this step we prove that for any $K>0$ sufficiently large, there exists a constant $\widetilde{\mathfrak{c}}_{\theta}=\widetilde{\mathfrak{c}}_{\theta}(K)>0$ such that

$$
\mathcal{N}_{-1 / 4-E}\left(\widetilde{Q}_{10}\right) \leq \widetilde{\mathfrak{c}}_{\theta}
$$

for all $E>0$. To do so, we introduce the quadratic form $\widetilde{Q}_{10}^{\prime}$ defined as

$$
\widetilde{Q}_{10}^{\prime}[u]:=\|\nabla u\|_{\widetilde{\Omega}_{10}}^{2}-\int_{K(\sin \theta)^{-1}}^{2 K(\sin \theta)^{-1}}|u(s, 0)|^{2} \mathrm{~d} s, \quad \operatorname{dom} \widetilde{Q}_{10}^{\prime}:=\operatorname{dom} \widetilde{Q}_{10} .
$$

One can check that the above form is closed, symmetric, densely defined and semibounded in $L^{2}\left(\widetilde{\Omega}_{10}\right)$. Now, for any $u \in \operatorname{dom} \widetilde{Q}_{10}$ we have

$$
\widetilde{Q}_{10}[u] \geq \widetilde{Q}_{10}^{\prime}[u]-K^{-2}\left(\|W\|_{\infty}+1 / 4\right)\|u\|_{\widetilde{\Omega}_{10}}^{2}
$$

Consequently, we get

$$
\mathcal{N}_{-1 / 4-E}\left(\widetilde{Q}_{10}\right) \leq \mathcal{N}_{-1 / 4-E+K^{-2}\left(\|W\|_{\infty}+1 / 4\right)}\left(\widetilde{Q}_{10}^{\prime}\right) .
$$

Next, we choose $K>0$ sufficiently large such that $-1 / 4+K^{-2}\left(\|W\|_{\infty}+1 / 4\right)<-1 / 8$. Combining (3.33) and (3.34) we have

$$
\mathcal{N}_{-1 / 4-E}\left(\widetilde{Q}_{10}\right) \leq \mathcal{N}_{-1 / 8}\left(\widetilde{Q}_{10}^{\prime}\right) .
$$

Then, let us split the domain $\widetilde{\Omega}_{10}$ into two disjoint sub-domains

$$
\widetilde{\Omega}_{100}=\left\{(s, t) \in \widetilde{\Omega}_{10}:|t|<1\right\}, \quad \widetilde{\Omega}_{101}=\left\{(s, t) \in \widetilde{\Omega}_{10}:|t|>1\right\} .
$$


We denote by $\widetilde{\Sigma}_{0}$ the image of $\Sigma_{0}$ under rotation (2.14) and, for $j=0,1$, let us define $\widetilde{\Sigma}_{0 j}=\widetilde{\Sigma}_{0} \cap \partial \Omega_{10 j}(j=0,1)$. Then, we consider the quadratic forms $\widetilde{Q}_{100}^{\prime}$ and $\widetilde{Q}_{101}^{\prime}$ defined as

$$
\begin{aligned}
\widetilde{Q}_{100}^{\prime}[u] & :=\|\nabla u\|_{\widetilde{\Omega}_{100}}^{2}-\int_{K(\sin \theta)^{-1}}^{2 K(\sin \theta)^{-1}}|u(s, 0)|^{2} \mathrm{~d} s, \\
\widetilde{Q}_{101}^{\prime}[u] & :=\|\nabla u\|_{\widetilde{\Omega}_{101}}^{2}, \\
\operatorname{dom} \widetilde{Q}_{10 j}^{\prime} & :=\left\{u \in H^{1}\left(\widetilde{\Omega}_{10 j}\right):\left.u\right|_{\widetilde{\Sigma}_{0 j}}=0\right\}, \quad j=0,1 .
\end{aligned}
$$

The above forms are closed, symmetric, densely defined and semibounded in $L^{2}\left(\widetilde{\Omega}_{100}\right)$ and in $L^{2}\left(\widetilde{\Omega}_{101}\right)$, respectively. For $u \in \operatorname{dom} \widetilde{Q}_{10}^{\prime}$, we define $u_{j}=\left.u\right|_{\widetilde{\Omega}_{10 j}}(j=0,1)$ and get

$$
\widetilde{Q}_{10}^{\prime}[u]=\widetilde{Q}_{100}^{\prime}\left[u_{0}\right]+\widetilde{Q}_{101}^{\prime}\left[u_{1}\right] .
$$

The above equality and the min-max principle yield

$$
\mathcal{N}_{-1 / 8}\left(\widetilde{Q}_{10}^{\prime}\right) \leq \mathcal{N}_{-1 / 8}\left(\widetilde{Q}_{100}^{\prime}\right)+\mathcal{N}_{-1 / 8}\left(\widetilde{Q}_{101}^{\prime}\right)
$$

For all $u \in \operatorname{dom} \widetilde{Q}_{101}^{\prime}, \widetilde{Q}_{101}^{\prime}[u] \geq 0$ and we get

$$
\mathcal{N}_{-1 / 8}\left(\widetilde{Q}_{101}^{\prime}\right)=0
$$

The quadratic form $\widetilde{Q}_{100}^{\prime}$ is the quadratic form of a Schrödinger operator with a $\delta$-interaction supported on a line segment. It acts on a bounded domain with mixed boundary conditions (Neumann and Dirichlet) thus, this operator has compact resolvent and its sequence of eigenvalues goes to infinity. Hence, we have

$$
\mathcal{N}_{-1 / 8}\left(\widetilde{Q}_{100}^{\prime}\right)=\widetilde{\mathfrak{c}}_{\theta}<\infty,
$$

where $\widetilde{\mathfrak{c}}_{\theta}=\widetilde{\mathfrak{c}}_{\theta}(K)>0$. Combining (3.35), (3.36), (3.37) and (3.38) we obtain (3.32).

Step 5. To conclude, inserting (3.24), (3.31) and (3.32) into (3.23) we get

$$
\begin{aligned}
\limsup _{E \rightarrow 0+} \frac{\mathcal{N}_{-1 / 4-E}\left(Q_{\Gamma_{\theta}}\right)}{|\ln E|} & \leq \limsup _{E \rightarrow 0+}\left(\frac{\mathcal{N}_{-1 / 4-E}\left(Q_{0}\right)}{|\ln E|}+\frac{\mathcal{N}_{-1 / 4-E}\left(Q_{1}\right)}{|\ln E|}\right) \\
& \leq \limsup _{E \rightarrow 0+}\left(\frac{\mathcal{N}_{-1 / 4-E}\left(\widetilde{Q}_{11}\right)}{|\ln E|}+\frac{\widehat{\mathfrak{c}}_{\theta}}{|\ln E|}+\frac{\widetilde{\mathfrak{c}}_{\theta}}{|\ln E|}\right)=\limsup _{E \rightarrow 0+} \frac{\mathcal{N}_{-1 / 4-E}\left(\widetilde{Q}_{11}\right)}{|\ln E|} .
\end{aligned}
$$

Finally, it remains to note that the form $\widetilde{Q}_{11}$ is the form $Q_{\Omega_{\theta}^{K}}$ in (3.10). This ends the proof of Lemma 3.5.

\section{APPENDIX A. QUADRATIC FORMS $Q_{\Gamma_{\theta}}^{[l]}$}

The aim of this appendix is to prove the following proposition about the forms $Q_{\Gamma_{\theta}}^{[l]}$ in (2.5) and the spaces $\mathcal{C}_{0}^{\infty}\left(\overline{\mathbb{R}_{+}^{2}}\right)$, $\mathcal{C}_{0,0}^{\infty}\left(\overline{\mathbb{R}_{+}^{2}}\right)$ defined in Notation 2.1 .

Proposition A.1. Let $d \geq 3, l \geq 0$ and the quadratic forms $Q_{\Gamma_{\theta}}^{[l]}$ be defined as in (2.5). Then the following statements hold:

(i) for $d=3$ and any $l>0, \mathcal{C}_{0,0}^{\infty}\left(\overline{\mathbb{R}_{+}^{2}}\right)$ is a form core for $Q_{\Gamma_{\theta}}^{[l]}$;

(ii) for $d=3$ and $l=0$ or $d \geq 4$ and $l \geq 0, \mathcal{C}_{0}^{\infty}\left(\overline{\mathbb{R}_{+}^{2}}\right)$ is a form core for $Q_{\Gamma_{\theta}}^{[l]}$.

Before proving Proposition A.1, we need to introduce a few notation. For any function $u \in L_{\text {cyl }}^{2}\left(\mathbb{R}^{d}\right)$ we denote by $\widetilde{u} \in L^{2}\left(\mathbb{R}^{d}\right)$ the function $\widetilde{u}\left(x_{1}, \ldots, x_{d}\right)=u(r, z, \phi)$ in the physical coordinates ( $c f$. the change of variables (2.1)). 
Let us fix the dimension $d \geq 3$ and $l \geq 0$. We choose $M>0$ large enough such that for any $\widetilde{u} \in \operatorname{dom} Q_{\mathcal{C}}$ and any $\widehat{u} \in \operatorname{dom} Q_{\Gamma_{\theta}}^{[l]}$ we have

$$
Q_{\mathcal{C}}[\widetilde{u}] \geq-M\|\widetilde{u}\|_{\mathbb{R}^{d}}^{2}, \quad Q_{\Gamma_{\theta}}^{[l]}[\widehat{u}] \geq-M\|\widehat{u}\|_{L^{2}\left(\mathbb{R}_{+}^{2} ; r^{d-2} \mathrm{~d} r \mathrm{~d} z\right)}^{2} .
$$

We introduce the following norms associated with the quadratic forms $Q_{\mathcal{C}}$ and $Q_{\Gamma_{\theta}}^{[l]}$ defined, for $\widetilde{u} \in \operatorname{dom} Q_{\mathcal{C}}$ and $\widehat{u} \in \operatorname{dom} Q_{\Gamma_{\theta}}^{[l]}$, by

$$
\|\widetilde{u}\|_{Q_{\mathcal{C}}}^{2}:=Q_{\mathcal{C}}[\widetilde{u}]+(M+1)\|\widetilde{u}\|_{\mathbb{R}^{d}}^{2}, \quad\|\widehat{u}\|_{Q_{\Gamma_{\theta}}^{[l]}}^{2}:=Q_{\Gamma_{\theta}}^{[l]}[\widehat{u}]+(M+1)\|\widehat{u}\|_{L^{2}\left(\mathbb{R}_{+}^{2} ; r^{d-2} \mathrm{~d} r \mathrm{~d} z\right)}^{2} .
$$

Now, we state three lemmas that are proven in the end of the appendix.

Lemma A.2. Let $d \geq 3$ and $l \geq 0$. Then the following set inclusions hold:

(i) for $d=3$ and any $l>0, \mathcal{C}_{0,0}^{\infty}\left(\overline{\mathbb{R}_{+}^{2}}\right) \subset \operatorname{dom} Q_{\Gamma_{\theta}}^{[l]}$;

(ii) for $d=3$ and $l=0$ or $d \geq 4$ and $l \geq 0, \mathcal{C}_{0}^{\infty}\left(\overline{\mathbb{R}_{+}^{2}}\right) \subset \operatorname{dom} Q_{\Gamma_{\theta}}^{[l]}$.

Lemma A.3. Let $d=3$ and $l>0$. Then the following set inclusion $\mathcal{C}_{0}^{\infty}\left(\overline{\mathbb{R}_{+}^{2}}\right) \cap \operatorname{dom} Q_{\Gamma_{\theta}}^{[l]} \subset \mathcal{C}_{0,0}^{\infty}\left(\overline{\mathbb{R}_{+}^{2}}\right)$ holds.

Lemma A.4. Let $d \geq 3, l \geq 0$ and $k \in\{1, \ldots, c(d, l)\}$. For any $u(r, z, \phi)=\widehat{u}(r, z) Y_{l, k}^{d-2}(\phi) \in H_{\text {cyl }}^{1}\left(\mathbb{R}^{d}\right)$ with $\widehat{u} \in L^{2}\left(\mathbb{R}_{+}^{2} ; r^{d-2} \mathrm{~d} r \mathrm{~d} z\right)$, there exists $\widehat{u}_{n} \in \mathcal{C}_{0}^{\infty}\left(\overline{\mathbb{R}_{+}^{2}}\right) \cap \operatorname{dom} Q_{\Gamma_{\theta}}^{[l]}$ such that

$$
\left\|\widehat{u}_{n}-\widehat{u}\right\|_{Q_{\Gamma_{\theta}}^{[l]}} \rightarrow 0, \quad n \rightarrow \infty \text {. }
$$

Now, we have all the tools to prove Proposition A.1.

Proof of Proposition A.1. Let $d \geq 3$, fix $l \in \mathbb{N}_{0}$ and $k \in\{1, \ldots, c(d, l)\}$. Let $\widehat{u} \in \operatorname{dom} Q_{\Gamma_{\theta}}^{[l]}$, we define $u(r, z, \phi)=$ $\widehat{u}(r, z) Y_{l, k}^{d-2}(\phi) \in L_{\text {cyl }}^{2}\left(\mathbb{R}^{d}\right)$. One can show by direct computations that

$$
\begin{aligned}
\left\|\partial_{r} u\right\|_{L_{\text {cyl }}^{2}\left(\mathbb{R}^{d}\right)}^{2}+\left\|\partial_{z} u\right\|_{L_{c y \mid}^{2}\left(\mathbb{R}^{d}\right)}^{2}+\left\|r^{-1} \nabla_{\mathbb{S}^{d-2}} u\right\|_{L_{\mathrm{cyl}}^{2}\left(\mathbb{R}^{d}\right)}^{2}=\left\|\partial_{r} \widehat{u}\right\|_{L^{2}\left(\mathbb{R}_{+}^{2} ; r^{d-2} \mathrm{~d} r \mathrm{~d} z\right)}^{2}+\left\|\partial_{z} \widehat{u}\right\|_{L^{2}\left(\mathbb{R}_{+}^{2} ; r^{d-2} \mathrm{~d} r \mathrm{~d} z\right)}^{2} \\
+l(l+d-3)\left\|r^{-1} \widehat{u}\right\|_{L^{2}\left(\mathbb{R}_{+}^{2} ; r^{d-2} \mathrm{~d} r \mathrm{~d} z\right)}^{2} .
\end{aligned}
$$

As $\widehat{u} \in \operatorname{dom} Q_{\Gamma_{\theta}}^{[l]}$ the right hand side is finite and hence $u \in H_{\text {cyl }}^{1}\left(\mathbb{R}^{d}\right)$. Now, thanks to Lemma A.4 we know that there exists a sequence $\widehat{u}_{n} \in \mathcal{C}_{0}^{\infty}\left(\overline{\mathbb{R}_{+}^{2}}\right) \cap \operatorname{dom} Q_{\Gamma_{\theta}}^{[l]}$ such that $\left\|\widehat{u}_{n}-\widehat{u}\right\|_{Q_{\Gamma_{\theta}}^{[l]}} \rightarrow 0$ as $n \rightarrow \infty$. Because $\widehat{u}_{n} \in \mathcal{C}_{0}^{\infty}\left(\overline{\mathbb{R}_{+}^{2}}\right)$ and thanks to Lemma A.2 (ii) we obtain item (ii) in Proposition A.1. To get item (i) in Proposition A.1, we use Lemma A.3, which yields $\widehat{u}_{n} \in \mathcal{C}_{0,0}^{\infty}\left(\overline{\mathbb{R}_{+}^{2}}\right)$. We conclude using Lemma A.2 (i).

Proof of Lemma A.2. By definition of the form domains in (2.5), it is only necessary to check that for $d=3, l>0$ and any $\widehat{u} \in \mathcal{C}_{0,0}^{\infty}\left(\overline{\mathbb{R}_{+}^{2}}\right)$ we have $r^{-1} \widehat{u} \in L^{2}\left(\mathbb{R}_{+}^{2} ; r \mathrm{~d} r \mathrm{~d} z\right)$. Let us fix $\widehat{u} \in \mathcal{C}_{0,0}^{\infty}\left(\overline{\mathbb{R}_{+}^{2}}\right)$, using a Taylor expansion in the $r$-variable, we can write $\widehat{u}(r, z)=r g(r, z)$ with $g \in \mathcal{C}_{0}^{\infty}\left(\overline{\mathbb{R}_{+}^{2}}\right)$. Consequently, we get

$$
\left\|r^{-1} \widehat{u}\right\|_{L^{2}\left(\mathbb{R}_{+}^{2} ; r \mathrm{~d} r \mathrm{~d} z\right)}=\|g\|_{L^{2}\left(\mathbb{R}_{+}^{2} ; r \mathrm{~d} r \mathrm{~d} z\right)}<\infty,
$$

which concludes the proof.

Proof of Lemma A.3. Let $d=3$ and $l>0$. We take $\widehat{u} \in \mathcal{C}_{0}^{\infty}\left(\overline{\mathbb{R}_{+}^{2}}\right) \cap \operatorname{dom} Q_{\Gamma_{\theta}}^{[l]}$ and, using a Taylor expansion in the $r$-variable, we can write $\widehat{u}(r, z)=\widehat{u}(0, z)+r g(r, z)$ with $g \in \mathcal{C}_{0}^{\infty}\left(\overline{\mathbb{R}_{+}^{2}}\right)$. Because $r^{-1} \widehat{u} \in L^{2}\left(\mathbb{R}_{+}^{2} ; r \mathrm{~d} r \mathrm{~d} z\right)$, we obtain that $r^{-1} \widehat{u}(0, z)=r^{-1} \widehat{u}(r, z)-g(r, z) \in L^{2}\left(\mathbb{R}_{+}^{2} ; r \mathrm{~d} r \mathrm{~d} z\right)$. Hence, we get

$$
\int_{\mathbb{R}_{+}^{2}} \frac{|\widehat{u}(0, z)|^{2}}{r} \mathrm{~d} r \mathrm{~d} z<\infty .
$$


Finiteness of the last integral necesserily implies $\widehat{u}(0, z)=0$ for any $z \in \mathbb{R}$. Hence, $\widehat{u}(r, z)=r g(r, z)$ and $\widehat{u} \in \mathcal{C}_{0,0}^{\infty}\left(\overline{\mathbb{R}_{+}^{2}}\right)$.

Proof of Lemma A.4. Let $d \geq 3, l \in \mathbb{N}_{0}$ and $k \in\{1, \ldots, c(d, l)\}$. Let the orthogonal projector $\Pi_{l, k}$ in $L_{\text {cyl }}^{2}\left(\mathbb{R}^{d}\right)$ be defined as in (2.3). Since $\widetilde{u} \in H^{1}\left(\mathbb{R}^{d}\right)$, there exists a sequence $\widetilde{v}_{n} \in \mathcal{C}_{0}^{\infty}\left(\mathbb{R}^{d}\right)$ such that $\left\|\widetilde{v}_{n}-\widetilde{u}\right\|_{H^{1}\left(\mathbb{R}^{d}\right)} \rightarrow 0$ as $n \rightarrow \infty$.

Further, define the modified sequence

$$
u_{n}:=\Pi_{l, k}\left(v_{n}\right) \in L_{\text {cyl }}^{2}\left(\mathbb{R}^{d}\right), \quad n \in \mathbb{N} .
$$

We remark that

$$
\widehat{u}_{n}(r, z):=\left\langle u_{n}(r, z, \cdot), Y_{l, k}^{d-2}(\cdot)\right\rangle_{\mathbb{S}^{d-2}} \in \mathcal{C}_{0}^{\infty}\left(\overline{\mathbb{R}_{+}^{2}}\right) .
$$

Thus, we can write $u_{n}(r, z, \phi)=\widehat{u}_{n}(r, z) Y_{l, k}^{d-2}(\phi) \in H_{\text {cyl }}^{1}\left(\mathbb{R}^{d}\right)$.

Next, we prove that

$$
\left\|u_{n}-u\right\|_{H_{\mathrm{cyl}}^{1}\left(\mathbb{R}^{d}\right)} \rightarrow 0, \quad n \rightarrow \infty
$$

By orthogonality, we have

$$
\left\|v_{n}-u\right\|_{L_{\text {cyl }}^{2}\left(\mathbb{R}^{d}\right)}^{2}=\left\|u_{n}-u\right\|_{L_{\text {cyl }}^{2}\left(\mathbb{R}^{d}\right)}^{2}+\left\|\left(I-\Pi_{k, l}\right) v_{n}\right\|_{L_{\text {cyl }}^{2}\left(\mathbb{R}^{d}\right)}^{2},
$$

where the left hand side tends to zero as $n \rightarrow \infty$. Hence, we get

$$
\left\|u_{n}-u\right\|_{L_{\text {cyl }}^{2}\left(\mathbb{R}^{d}\right)} \rightarrow 0, \quad n \rightarrow \infty
$$

Because $v_{n}$ is smooth and compactly supported, we have the following useful commutation relations

$$
\partial_{r} u_{n}=\Pi_{l, k}\left(\partial_{r} v_{n}\right) \quad \text { and } \quad \partial_{z} u_{n}=\Pi_{l, k}\left(\partial_{z} v_{n}\right)
$$

which yield

$$
\begin{aligned}
& \left\|\partial_{r}\left(u_{n}-u\right)\right\|_{L_{\mathrm{cyl}}^{2}\left(\mathbb{R}^{d}\right)}=\left\|\Pi_{l, k}\left(\partial_{r}\left(v_{n}-u\right)\right)\right\|_{L_{\mathrm{cyl}}^{2}\left(\mathbb{R}^{d}\right)} \leq\left\|\partial_{r}\left(v_{n}-u\right)\right\|_{L_{\mathrm{cyl}}^{2}\left(\mathbb{R}^{d}\right)} \rightarrow 0, \quad n \rightarrow \infty, \\
& \left\|\partial_{z}\left(u_{n}-u\right)\right\|_{L_{\text {cyl }}^{2}\left(\mathbb{R}^{d}\right)}=\left\|\Pi_{l, k}\left(\partial_{z}\left(v_{n}-u\right)\right)\right\|_{L_{\text {cyl }}^{2}\left(\mathbb{R}^{d}\right)} \leq\left\|\partial_{z}\left(v_{n}-u\right)\right\|_{L_{\text {cyl }}^{2}\left(\mathbb{R}^{d}\right)} \rightarrow 0, \quad n \rightarrow \infty .
\end{aligned}
$$

Using that spherical harmonics are eigenfunctions of the self-adjoint Laplace-Beltrami operator $-\Delta_{\mathbb{S}^{d-2}}$ associated with the quadratic form $H^{1}\left(\mathbb{S}^{d-2}\right) \ni \psi \mapsto\left\|\nabla_{\mathbb{S}^{d-2}} \psi\right\|_{\mathbb{S}^{d-2}}^{2}$ on $L^{2}\left(\mathbb{S}^{d-2}\right)$, we have for any fixed $(r, z) \in \mathbb{R}_{+}^{2}$

$$
\begin{aligned}
\left\langle\nabla_{\mathbb{S}^{d-2}}\left(u_{n}-u\right), \nabla_{\mathbb{S}^{d-2}}\left(v_{n}-u_{n}\right)\right\rangle_{\mathbb{S}^{d-2}} & =\left\langle\left(\widehat{u}_{n}-\widehat{u}\right)\left(-\Delta_{\mathbb{S}^{d-2}} Y_{l, k}^{d-2}\right),\left(I-\Pi_{l, k}\right) v_{n}\right\rangle_{\mathbb{S}^{d-2}} \\
& =l(l+d-3)\left\langle u_{n}-u,\left(I-\Pi_{l, k}\right) v_{n}\right\rangle_{\mathbb{S}^{d-2}} \\
& =l(l+d-3)\left\langle\Pi_{l, k}\left(v_{n}-u_{n}\right),\left(I-\Pi_{l, k}\right) v_{n}\right\rangle_{\mathbb{S}^{d-2}}=0 .
\end{aligned}
$$

The above relation gives

$$
\left\|\nabla_{\mathbb{S}^{d-2}}\left(v_{n}-u\right)\right\|_{\mathbb{S}^{d-2}}^{2}=\left\|\nabla_{\mathbb{S}^{d}-2}\left(u_{n}-u\right)\right\|_{\mathbb{S}^{d-2}}^{2}+\left\|\nabla_{\mathbb{S}^{d-2}}\left(v_{n}-u_{n}\right)\right\|_{\mathbb{S}^{d-2}}^{2} .
$$

Multiplying the latter equality by $r^{-2}$ and integrating in $(r, z)$ we get

$$
\left\|r^{-1} \nabla_{\mathbb{S}^{d-2}}\left(u_{n}-u\right)\right\|_{L_{\mathrm{cyl}}^{2}\left(\mathbb{R}^{d}\right)}^{2} \leq\left\|r^{-1} \nabla_{\mathbb{S}^{d-2}}\left(v_{n}-u\right)\right\|_{L_{\mathrm{cyl}}^{2}\left(\mathbb{R}^{d}\right)}^{2} \leq\left\|v_{n}-u\right\|_{H_{\mathrm{cyl}}^{1}\left(\mathbb{R}^{d}\right)}^{2} \rightarrow 0, \quad n \rightarrow \infty .
$$

Finally, combining (A.2), (A.3), (A.4) and the fact that $u \in H_{\text {cyl }}^{1}\left(\mathbb{R}^{d}\right)$ we get (A.1).

According to [2, Prop. 3.1 and its proof] and Notation 2.1, the $H_{\text {cyl }}^{1}\left(\mathbb{R}^{d}\right)$-norm is equivalent to the norm $\|\cdot\|_{Q_{\mathcal{C}}}$ after suitable identification of functions. Hence, (A.1) yields

$$
\left\|\widetilde{u}_{n}-\widetilde{u}\right\|_{Q_{c}} \rightarrow 0, \quad n \rightarrow \infty .
$$

By direct computation, we get

$$
\left\|\widetilde{u}_{n}-\widetilde{u}\right\|_{Q_{\mathcal{C}}}=\left\|\widehat{u}_{n}-\widehat{u}\right\|_{Q_{\Gamma_{\theta}}^{[l]}},
$$

which concludes the proof of Lemma A.4. 
Acknowledgements. V. Lotoreichik is supported by the Czech Science Foundation (GAČR) under the project 14-06818S. He is grateful for the stimulating research stay and the hospitality at the Basque Center for Applied Mathematics in May 2015 where a part of this paper was written. T. Ourmières-Bonafos is supported by the Basque Government through the BERC 2014-2017 program and by Spanish Ministry of Economy and Competitiveness MINECO: BCAM Severo Ochoa excellence accreditation SEV-2013-0323.

\section{REFERENCES}

[1] S. Albeverio, F. Gesztesy, R. Høegh-Krohn, and H. Holden. Solvable models in quantum mechanics. AMS Chelsea Publishing, Providence, RI, second edition, 2005. With an appendix by Pavel Exner.

[2] J. Behrndt, P. Exner, and V. Lotoreichik. Schrödinger operators with $\delta$ - and $\delta^{\prime}$-interactions on Lipschitz surfaces and chromatic numbers of associated partitions. Rev. Math. Phys., 26(8):43, 2014.

[3] J. Behrndt, P. Exner, and V. Lotoreichik. Schrödinger operators with $\delta$-interactions supported on conical surfaces. J. Phys. A, Math. Theor, 47(35):16, 2014.

[4] C. Bernardi, M. Dauge, and Y. Maday. Spectral methods for axisymmetric domains, volume 3 of Series in Applied Mathematics (Paris). Gauthier-Villars, Éditions Scientifiques et Médicales Elsevier, Paris, 1999. Numerical algorithms and tests due to Mejdi Azaïez.

[5] V. Bonnaillie-Noël, M. Dauge, and N. Popoff. Ground state energy of the magnetic Laplacian on general three-dimensional corner domains. ArXiv e-prints, Mar. 2014.

[6] J. F. Brasche, P. Exner, Y. A. Kuperin, and P. Šeba. Schrödinger operators with singular interactions. J. Math. Anal. Appl., 184(1):112-139, 1994.

[7] B. Brown, M. Eastham, and I. Wood. An example on the discrete spectrum of a star graph. In Analysis on graphs and its applications. Selected papers based on the Isaac Newton Institute for Mathematical Sciences programme, Cambridge, UK, January 8-June 29, 2007, pages 331-335. Providence, RI: American Mathematical Society (AMS), 2008.

[8] R. Brummelhuis and P. Duclos. Effective Hamiltonians for atoms in very strong magnetic fields. J. Math. Phys., 47(3):032103, $41,2006$.

[9] V. Bruneau and N. Popoff. On the negative spectrum of the Robin Laplacian in corner domains. ArXiv e-prints, Nov. 2015.

[10] H. L. Cycon, R. G. Froese, W. Kirsch, and B. Simon. Schrödinger operators with application to quantum mechanics and global geometry. Texts and Monographs in Physics. Springer-Verlag, Berlin, study edition, 1987.

[11] M. Dauge, Y. Lafranche, and N. Raymond. Quantum waveguides with corners. ESAIM, Proc., 35:14-45, 2012.

[12] M. Dauge, T. Ourmières-Bonafos, and N. Raymond. Spectral asymptotics of the Dirichlet Laplacian in a conical layer. Commun. Pure Appl. Anal., 14(3):1239-1258, 2015.

[13] A.-S. B.-B. Dhia, S. Fliss, C. Hazard, and A. Tonnoir. A Rellich type theorem for the Helmholtz equation in a conical domain. Comptes Rendus Mathematique, 354(1):27-32, 2016.

[14] V. Duchêne and N. Raymond. Spectral asymptotics of a broken $\delta$-interaction. J. Phys. A, Math. Theor., 47(15):19, 2014.

[15] P. Exner. Leaky quantum graphs: a review. In Analysis on graphs and its applications, volume 77 of Proc. Sympos. Pure Math., pages 523-564. Amer. Math. Soc., Providence, RI, 2008.

[16] P. Exner and T. Ichinose. Geometrically induced spectrum in curved leaky wires. J. Phys. A, Math. Gen., 34(7):1439-1450, 2001.

[17] P. Exner and S. Kondej. Bound states due to a strong $\delta$ interaction supported by a curved surface. J. Phys. A, Math. Gen., 36(2):443-457, 2003.

[18] P. Exner and S. Kondej. Gap asymptotics in a weakly bent leaky quantum wire. ArXiv e-prints, June 2015.

[19] P. Exner and H. Kovař́ík. Quantum waveguides. Theoretical and Mathematical Physics. Springer, Cham, 2015.

[20] P. Exner and K. Němcová. Leaky quantum graphs: Approximations by point-interaction Hamiltonians. J. Phys. A, Math. Theor., 36(40):1017310193, 2003.

[21] P. Exner and M. Tater. Spectrum of Dirichlet Laplacian in a conical layer. J. Phys. A, Math. Theor., 43(47):11, 2010.

[22] P. Exner and K. Yoshitomi. Asymptotics of eigenvalues of the Schrödinger operator with a strong $\delta$-interaction on a loop. J. Geom. Phys., 41(4):344-358, 2002.

[23] A. Figotin and P. Kuchment. Band-gap structure of spectra of periodic dielectric and acoustic media. II: Two-dimensional photonic crystals. SIAM J. Appl. Math., 56(6):1561-1620, 1996.

[24] A. Hassell and S. Marshall. Eigenvalues of Schrödinger operators with potential asymptotically homogeneous of degree -2 . Trans. Am. Math. Soc., 360(8):4145-4167, 2008.

[25] T. Kato. Perturbation theory for linear operators. Reprint of the corr. print. of the 2nd ed. 1980. Berlin: Springer-Verlag, reprint of the corr. print. of the 2nd ed. 1980 edition, 1995.

[26] W. Kirsch and B. Simon. Corrections to the classical behavior of the number of bound states of Schrödinger operators. Ann. Phys., 183(1):122-130, 1988.

[27] A. Kostenko and M. Malamud. 1-D Schrödinger operators with local point interactions: a review. In Spectral analysis, differential equations and mathematical physics: A Festschrift in honor of Fritz Gesztesy's 60th birthday, pages 235-262. Providence, RI: American Mathematical Society (AMS), 2013.

[28] K. Pankrashkin. On the discrete spectrum of Robin Laplacians in conical domains. to appear in Math. Model. Nat. Phenom., ArXiv e-prints, July 2015.

[29] M. Reed and B. Simon. Methods of modern mathematical physics. IV. Analysis of operators. Academic Press, New York, 1978.

[30] K. Schmüdgen. Unbounded self-adjoint operators on Hilbert space. Dordrecht: Springer, 2012. 
[31] E. M. Stein and G. Weiss. Introduction to Fourier analysis on Euclidean spaces. Princeton Mathematical Series. Princeton, N. J.: Princeton University Press. X, 297 p., 1971.

[32] J. Weidmann. Lineare Operatoren in Hilberträumen. Teil I: Grundlagen. Wiesbaden: B. G. Teubner, 2000.

Department of Theoretical Physics, Nuclear Physics Institute, Czech Academy of Sciences, 250 68, Řež near Prague, CZECH REPUBlic

E-mail address: lotoreichik@ujf.cas.cz

BCAM - Basque Center for Applied Mathematics, Alameda de Mazarredo, 14 E48009 Bilbao, Basque Country - Spain

E-mail address: tourmieres@bcamath.org 